\title{
Research Article \\ Performance Capabilities of Long-Range UWB-IR TDOA Localization Systems
}

\author{
Richard J. Barton' ${ }^{1}$ and Divya Rao² \\ ${ }^{1}$ Engineering Research and Consulting, Inc., NASA Johnson Space Center, Houston, TX 77058, USA \\ ${ }^{2}$ Cisco Systems, Inc., San Jose, CA 95134, USA \\ Correspondence should be addressed to Richard J. Barton, richard.j.barton@nasa.gov
}

Received 11 March 2007; Accepted 26 October 2007

Recommended by Venugopal V. Veeravalli

The theoretical and practical performance limits of a 2D ultra-wideband impulse-radio localization system operating in the far field are studied under the assumption that estimates of location are based on time-difference-of-arrival (TDOA) measurements. Performance is evaluated in the presence of errors in both the TDOA measurements and the sensor locations. The performance of both optimal (maximum-likelihood) and suboptimal location estimation algorithms is studied and compared with the theoretical performance limit defined by the Cramér-Rao lower bound on the variance of unbiased TDOA location estimates. A novel weighted total-least-squares algorithm is introduced that compensates somewhat for errors in sensor positions and reduces the bias in location estimation compared with a widely used weighted least-squares approach. In addition, although target tracking per se is not considered in this paper, performance is evaluated both under the assumption that sequential location estimates are not aggregated as well as under the assumption that some sort of tracker is available to aggregate a sequence of estimates.

Copyright (c) 2008 R. J. Barton and D. Rao. This is an open access article distributed under the Creative Commons Attribution License, which permits unrestricted use, distribution, and reproduction in any medium, provided the original work is properly cited.

\section{INTRODUCTION}

Ultra-wideband (UWB) impulse radio (IR) technology is a high-bandwidth communication scheme that offers several advantages for location estimation of targets based on radiofrequency emissions. In particular, the bandwidth of UWBIR signals is on the order of several gigahertz $(\mathrm{GHz})$, which translates to a time resolution in the subnanosecond range. As a result of this fine time resolution, UWB-IR transmissions are well suited for precise positioning using time domain techniques. In addition, the wide bandwidth of the signals results in very low power spectral densities, which reduces interference on other RF systems, and the short pulse duration reduces or eliminates pulse distortion (fading) and spurious signal detections due to multipath propagation.

In this paper, the theoretical and practical performance limits of a 2D UWB-IR time-difference-of-arrival (TDOA) localization system are studied. For purposes of this work, we assume that the target is in the far field in the sense that the range of the target is always much greater than the radius of the smallest circle containing all of the receiving sensors. Performance is evaluated in the presence of errors in both the TDOA measurements and in the sensor position measurements. In addition, although target tracking per se is not considered in this paper, performance is evaluated both under the assumption that sequential location estimates are not aggregated (i.e., one-shot location estimation) and under the assumption that some sort of tracker is available to aggregate a sequence of location estimates. For the second scenario, simple block averaging of individual location estimates for a stationary target is adopted to simulate the behavior of a tracker operating with a moving target.

It should be noted that consideration of the impact of a tracker and errors in sensor positions is particularly important for performance evaluation of UWB-IR localization systems, and both of these issues have so far been largely ignored in studies of such systems [1-4]. The impact of a tracker on the performance of the system is of interest because UWB systems of all types are constrained by FCC regulations to operate at very low power [5]. Hence, for tracking targets in the far field (e.g., at ranges exceeding 100-200 meters for a small sensor array), the signal-to-noise ratio (SNR) for a single received UWB-IR pulse will generally be quite low. If the target is either stationary or moving slowly relative to the 
pulse repetition rate (which can be in the megahertz range), consecutive pulses can be coherently averaged at the receiving sensors to increase the SNR and improve the accuracy of the location estimates. However, if the target is moving with moderate velocity, coherent pulse averaging over a period of sufficient duration to increase the SNR to the desired level will degrade the accuracy of the location estimates. Hence, in these situations, it is necessary to aggregate the sequence of location estimates noncoherently using a tracker. The difficulty here is that while such noncoherent averaging will effectively reduce the variance of the aggregate location estimate, any bias in the original low-SNR location estimates will not be removed. In such situations, the error floor introduced by the bias will determine the asymptotic accuracy of the localization system.

In addition, the impact of errors in sensor positions on localization performance is of particular interest for UWBIR systems precisely because of the extreme precision that such systems are theoretically capable of delivering. That is, the large bandwidth and short duration of a UWB-IR pulse means that it is possible with only moderate SNR to measure the difference in time of arrival between pairs of pulses with a resolution in the $10-100$ picoseconds range. Since this corresponds to a distance-difference resolution on the order of a few centimeters, it is clear that sensor positions errors of only a few centimeters can become a dominant source of error in the final location estimate. Hence, the localization performance of a UWB-IR system can be severely impacted by relatively small errors in sensor position, and such impact must be considered in a comprehensive performance evaluation.

To determine the theoretical performance limits of a UWB-IR localization system in this paper, the Cramér-Rao lower bound (CRLB) for the variance of unbiased TDOA estimates has been evaluated under the assumption that the waveform transmitted from the target satisfies a root-meansquare bandwidth constraint and that the channels of the receiving sensors are well modeled as band-limited additive white Gaussian noise (AWGN) channels. In addition, the CRLB for unbiased estimates of location in 2D based on inaccurate TDOA measurements has been derived under the assumption that the sensor positions are known precisely and that the TDOA measurement errors are independent identically distributed (i.i.d.) zero-mean Gaussian random variables with variance determined by the CRLB for TDOA estimation. Taken in conjunction, these two bounds provide a lower bound on the achievable performance of a 2D UWBIR TDOA localization and tracking system in the absence of errors in sensor position measurements, subject to the constraints imposed by other system parameters such as SNR, number of receiving sensors, target range, and sensor geometry. ${ }^{1}$ As we discuss below, under reasonable simplifying as-

\footnotetext{
${ }^{1}$ Recent work on improved lower bounds for time-of-arrival estimation error in UWB channels $[6,7]$ indicates that tighter lower bounds on system performance may be available using the Ziv-Zakai lower bound (ZZLB) rather than the CRLB in realistic UWB systems. However, since the ZZLB requires information regarding the autocorrelation function of the transmitted pulse, the more straightforward CRLB is used in this paper.
}

sumptions, the CRLB for unbiased estimates of location in the presence of sensor position errors as well as TDOA measurement errors takes the same form as the CRLB with only TDOA measurement errors. Hence, we are able to use the same bound (with an appropriate adjustment for the increase in aggregate measurement error variance) as a lower bound on the achievable performance both with and without sensor position errors in some cases.

To determine the more practical performance limits of UWB-IR localization and compare these with the theoretical limits presented in the paper, three different location estimation algorithms have been explored to solve the system of hyperbolic equations defined by the TDOA problem. The algorithms studied are identified throughout the paper as the weighted minimum least-squares (WMLS) technique, the weighted total least-squares (WTLS) technique, and the Newton-Raphson method (N-R). The WMLS approach uses a constrained linear model described in [8] to compute an estimate of the target location. The WTLS is a novel modification of the WMLS method that compensates for the errors in sensor positions as well as in the TDOA measurements. The N-R method is equivalent to an approximate maximumlikelihood (ML) technique, in which the nonlinear system of TDOA equations is solved iteratively, using the location estimate provided by the WMLS algorithm as a starting value for the iteration.

The performance of the three baseline algorithms was evaluated using Monte Carlo simulations. Scenarios with and without errors in sensor positions were considered. The performance metrics evaluated and compared were the bias and mean-squared error (MSE) of both one-shot and averaged location estimates. The theoretical values of the bias and MSE were also derived analytically for the WMLS algorithm and used both to validate the simulation results and provide a theoretical baseline for performance evaluation.

The contributions of this work can be summarized as follows:

(i) Most of the previous work on UWB-IR location estimation has focused on target localization in the near field; that is, when the target range is on the order of the radius of the smallest circle containing all of the receiving sensors. Such studies are sufficient for many UWB applications, but UWB-IR localization can also be applied effectively in the far field and such systems are currently being developed for some applications $[9,10]$. For the near-field case, SNR is often high and system performance is relatively insensitive to changes in tracking geometry caused by small sensor position errors or small changes in range. On the other hand, non-line-of-sight (NLOS) and multipath propagation are often prevalent (particularly for indoor environments), difficult to discriminate from line-of-sight (LOS) propagation, and can cause serious performance degradation. In the far-field case, the situation is somewhat reversed, particularly for outdoor applications such as those considered in $[9,10]$. SNR can be quite low and system performance is extremely sensitive to both small sensor position errors and relatively small changes in range, but NLOS and 
multipath propagation are often much less of a concern. Hence, in this work, we have neglected the effects of NLOS and multipath propagation and concentrated instead on the effects of low SNR, range, and errors in sensor position. The results presented in this paper provide a comprehensive evaluation of the theoretical and practical performance characteristics of UWB-IR TDOA localization systems operating in the far field. An attempt has been made to present sufficient detail and breadth in the performance evaluations that the results presented in this paper may prove useful for practical system design.

(ii) We demonstrate that in low-SNR situations where one-shot location estimates must be averaged using a tracker in order to produce final location estimates of acceptable accuracy, extreme care must be taken to utilize an estimation algorithm that is as close to unbiased as possible. In particular, we show that the relatively small one-shot bias present in the most commonly used constrained least-squares TDOA algorithm rapidly dominates the overall MSE of the location estimates in the low-SNR regime when a moderate amount of block-averaging is performed on the sequence of estimates. We also demonstrate that this bias can be greatly reduced without substantially increasing the complexity of the location estimation algorithms. In particular, we derive a new weighted totalleast-squares algorithm and show that both it and a standard maximum-likelihood approach have much smaller bias in the low-SNR regime with very little increase in complexity.

(iii) Finally, we consider the effect of sensor position errors on the performance of UWB-IR location estimation. We demonstrate that relatively small sensor position errors that are quite likely to occur in practice can seriously bias the location estimates produced by algorithms that make no attempt to account for them. We also demonstrate that this bias cannot be readily eliminated, as in the low-SNR regime discussed above, by resorting to simple low-complexity algorithmic modifications such as total-least-squares or maximum-likelihood approaches.

The remainder of this paper is organized as follows. Section 2 discusses the relationship of the current work with other recent work on UWB location estimation. Section 3 provides some background on TDOA location estimation algorithms and discusses the three localization algorithms studied in this work. The performance analysis is summarized in Section 4, including the results on the statistical performance characteristics of the WMLS algorithm, discussion of the two CRLBs utilized in the paper, description of the Monte-Carlo simulations, and the results of the performance evaluation are presented. Section 5 discusses the implications of the performance results and presents some concluding remarks.

\section{RELATIONSHIP WITH PREVIOUS WORK}

Various aspects of the problem of target localization using UWB-IR signals have been studied recently by different au- thors. In this section, we briefly review some of these studies and discuss how they relate to the results presented in this paper.

In [1], Gezici et al. discuss positioning techniques based on time-of-arrival (TOA), direction-of-arrival (DOA), and received signal strength (RSS), along with their feasibility for use in UWB-IR systems. Theoretical limits on TOA estimation and sources of error are explored in detail, and new approaches for low-complexity TOA estimation in dense multipath environments and hybrid RSS-TOA location estimation are discussed. The emphasis in [1] is primarily on TOA approaches to location estimation. The performance of TDOA techniques, which are the focus of the current paper, is largely ignored.

The study in [11] investigates object tracking in a $2 \mathrm{D}$ UWB-IR sensor network using multipath measurements in different scenarios: a single transmitter with a single receiver, multiple transmitters with a single receiver, and multiple transmitters with multiple receivers. The study is not specific to any localization algorithm, but computes the CRLB for the high-SNR case in each of the above scenarios. In the third scenario, the additional process of sorting multipath arrivals between different sensor pairs into sets corresponding to a single physical object has been considered. The work in [11] is particularly relevant for UWB-IR localization scenarios in which there is either no LOS component or for which the LOS component cannot be clearly differentiated from the NLOS components of the received signal. In this paper, we focus on the scenario in which the LOS component of the signal is not distorted by NLOS or multipath components, and localization is performed solely on the basis of LOS signals. The effects of multipath propagation have been ignored under the assumption that the LOS path can be reliably detected and discriminated from the later multipath arrivals.

In [3], the authors explore the use of TDOA location estimation techniques in conjunction with UWB-IR signals. A novel method for combining TDOA estimates from multiple antenna pairs to produce a final estimate of target location is introduced and studied via experimentation in a controlled environment. The primary emphasis in [3] is on reducing the variance in the TDOA estimates themselves in order to improve localization accuracy. In this paper, we assume that the TDOA estimates themselves are optimal (i.e., unbiased estimates that attain the CRLB) and focus on understanding the effects of SNR, range, sensor geometry, and sensor position errors on localization performance.

Other recent UWB-IR localization studies of interest include $[2,4,6,7,12-16]$; however, these studies are less closely related to the current paper than the three discussed above. The results in [2] relate entirely to targets in the near field and deal primarily with target detection rather than localization. The results in [4] are restricted to TOA rather than TDOA techniques as are the results in $[15,16]$, which also consider NLOS and multipath propagation. Monte Carlo localization in dense multipath UWB environments is considered in [14]. The CRLBs for synchronization and time-delay of UWB signals are studied in detail in $[12,13]$, and ZZLBs are studied in $[6,7]$. Given a particular system with a known pulse shape, such as a Gaussian monocycle, the theoretical performance 
bounds given in this paper could possibly be improved by employing the ZZLB rather than the CRLB.

\section{TDOA LOCALIZATION ALGORITHMS}

The principle of using TDOA measurements to perform localization has been widely studied in the literature. In this section, we give some background on the three TDOA localization algorithms studied in this paper and discuss their implementation.

One of the most well-known approaches, which is related to earlier work by Smith and Abel [17], was introduced by Chan and Ho in [8]. In this approach, the TDOA equations are solved using a two-stage, constrained, weighted linear least-squares technique. The technique is not iterative and does not suffer from convergence problems in the absence of a good initial condition as other linearized approaches often do. The performance of this estimation algorithm was also studied in [8] under the assumption that the sensor positions were known precisely and that the TDOA errors were small enough that the inherent bias of the approach was negligible. Both near-field and far-field target ranges were studied and it was shown that the CRLB for one-shot location estimation was approximately achieved in the high SNR regime studied.

Due to its low computational complexity, lack of convergence problems, and near optimal performance in high SNR situations, variations of the Chan and Ho algorithm are still the most widely utilized and studied among TDOA location estimation techniques. As such, we have adopted this approach as the baseline for our evaluation of the performance of practical UWB-IR localization and tracking systems. The main drawback of this approach is that it is not unbiased even in the absence of sensor position errors. Since a systematic bias cannot be removed by the operation of a conventional tracker, which nevertheless will very effectively reduce the variance in the resulting sequence of location estimates even if the target is moving, this bias will quickly dominate the performance in some situations. As a result, alternatives to this algorithm must be considered when evaluating the performance of UWB-IR localization systems.

The WMLS algorithm implemented and studied in this paper is identical to the Chan and Ho algorithm; however, in this study, both errors in sensor position and the effect of the bias in the algorithm have been considered. To reduce the bias of the WMLS algorithm, and to some extent its sensitivity to errors in sensor position, we have also modified the algorithm to compute location estimates using a novel weighted, constrained, total-least-squares approach. ${ }^{2}$ The details regarding the implementation of the WTLS algorithm are given below. For the same reasons, we have

\footnotetext{
${ }^{2}$ The advantage of the total-least-squares (TLS) approach in this case is that the bias of the solution to a TLS problem is generally smaller than the solution to the corresponding least-squares (LS) problem when there are unknown errors in the observation model [18]. The disadvantage is that the variance of the solution generally increases as the bias decreases, but in our case, that is a desirable tradeoff. The novelty the TLS algorithm introduced here is the addition of a weighting matrix analogous to the weighting matrix used in a conventional weighted LS approach.
}

also studied an approximate maximum-likelihood algorithm for position estimation based on TDOA measurements corrupted by additive white Gaussian noise (AWGN). In this case, an approximate solution for the likelihood equation is identified using the Newton-Raphson iterative method starting from the WMLS solution. The details regarding implementation of the N-R algorithm are also given below.

A recent study by Kovaviasaruch and Ho [19] presents an algebraic solution for estimating the position of an emitter based on TDOA measurements from an arbitrary array of sensors with random errors in the sensor position measurements. The proposed method was found to be computationally attractive and did not suffer from convergence or initialization problems. A subsequent paper [20] by the same authors presented an iterative algorithm for estimating the location of an emitter and the positions of the receivers simultaneously using TDOA measurements. The proposed method was based on Taylor-series expansions and suffered from poor convergence if a good initial solution was not available. Although these and other similar approaches are quite promising for application in UWB-IR systems in the presence of sensor position errors, they have not yet been widely utilized or studied, and we have not included them in our performance evaluations here.

\subsection{Implementation of the WMLS algorithm}

Throughout the remainder of this paper, we assume that there is one transmitter located at an unknown location $\left(x_{0}, y_{0}\right)$ in a two-dimensional space and that there are $M+1$ receivers, with one receiver at the origin and $M$ receivers located symmetrically in a circle around it. This particular geometry was chosen for the applications considered in [9] that motivated much of the current study; however, it has the additional advantage that the location estimation performance becomes isotropic as $M$ becomes large. The true receiver positions are given by $\left\{(0,0),\left(x_{1}, y_{1}\right),\left(x_{2}, y_{2}\right), \ldots,\left(x_{M}, y_{M}\right)\right\}$, and the true relative time delays between the arrival of the transmitted signal at receiver $(0,0)$ and each of the other locations $\left(x_{1}, y_{1}\right), \ldots,\left(x_{M}, y_{M}\right)$ are given by $\left\{\tau_{1}, \tau_{2}, \ldots, \tau_{M}\right\}$.

If the propagation velocity of the signals is given by the constant $c$, the relative time delays can be translated into distance differences that satisfy the following constrained system of linear equations in the absence of errors in the TDOA measurements or sensor positions:

$$
\mathbf{G}_{0} \mathbf{u}_{0}=\mathbf{h}_{0}
$$

where

$$
\mathbf{G}_{0}=-2 \cdot\left[\begin{array}{ccc}
x_{1} & y_{1} & c \tau_{1} \\
x_{2} & y_{2} & c \tau_{2} \\
\vdots & \vdots & \vdots \\
x_{M} & y_{M} & c \tau_{M}
\end{array}\right], \quad \mathbf{u}_{0}=\left[\begin{array}{c}
x_{0} \\
y_{0} \\
r_{0}
\end{array}\right]
$$




$$
\mathbf{h}_{0}=\left[\begin{array}{c}
c^{2} \tau_{1}^{2}-x_{1}^{2}-y_{1}^{2} \\
c^{2} \tau_{2}^{2}-x_{2}^{2}-y_{2}^{2} \\
\vdots \\
c^{2} \tau_{M}^{2}-x_{M}^{2}-y_{M}^{2}
\end{array}\right]
$$

and $r_{0}=\sqrt{x_{0}^{2}+y_{0}^{2}}$.

The system of equations has been made linear by introducing a third variable $r_{0}$, which represents the range of the transmitter from the origin. The solution of this constrained system of equations is found using a two-state weighted minimum least-squares approach. The first stage involves solving the linear system of equations, and the second stage refines the estimate obtained in stage 1 by enforcing the nonlinear constraint. This two-stage procedure, which was proposed by Chan and Ho in [8], is developed below.

In the presence of additive noise in the sensor positions and TDOA measurements, (1) becomes

$$
\mathbf{G}_{1} \mathbf{u}_{0}=\mathbf{h}_{1}-\left(\Delta \mathbf{h}_{1}-\Delta \mathbf{G}_{1} \mathbf{u}_{0}\right),
$$

where

$$
\begin{aligned}
\mathbf{G}_{1}= & \mathbf{G}_{0}+\Delta \mathbf{G}_{1}, \\
\mathbf{h}_{1}= & \mathbf{h}_{0}+\Delta \mathbf{h}_{1}, \\
\Delta \mathbf{G}_{1}= & -2 \cdot\left[\begin{array}{ccc}
\varepsilon_{11} & \varepsilon_{12} & c \delta_{1} \\
\varepsilon_{21} & \varepsilon_{22} & c \delta_{2} \\
\vdots & \vdots & \vdots \\
\varepsilon_{M 1} & \varepsilon_{M 2} & c \delta_{M}
\end{array}\right], \\
\Delta \mathbf{h}_{1}= & {\left[\begin{array}{c}
c^{2} \delta_{1}^{2}+2 c^{2} \tau_{1} \delta_{1}-2 x_{1} \varepsilon_{11}-2 y_{1} \varepsilon_{12}-\varepsilon_{11}^{2}-\varepsilon_{12}^{2} \\
c^{2} \delta_{2}^{2}+2 c^{2} \tau_{2} \delta_{2}-2 x_{2} \varepsilon_{21}-2 y_{2} \varepsilon_{22}-\varepsilon_{21}^{2}-\varepsilon_{22}^{2} \\
\vdots \\
c^{2} \delta_{M}^{2}+2 c^{2} \tau_{M} \delta_{M}-2 x_{M} \varepsilon_{M 1}-2 y_{M} \varepsilon_{M 2}-\varepsilon_{M 1}^{2}-\varepsilon_{M 2}^{2}
\end{array}\right] . }
\end{aligned}
$$

The vectors

$$
\begin{aligned}
\boldsymbol{\varepsilon}_{1} & =\left[\begin{array}{llll}
\varepsilon_{11} & \varepsilon_{21} & \cdots & \varepsilon_{M 1}
\end{array}\right]^{T}, \\
\boldsymbol{\varepsilon}_{2} & =\left[\begin{array}{llll}
\varepsilon_{12} & \varepsilon_{22} & \cdots & \varepsilon_{M 2}
\end{array}\right]^{T} \\
\boldsymbol{\delta} & =\left[\begin{array}{llll}
\delta_{1} & \delta_{2} & \cdots & \delta_{M}
\end{array}\right]^{T}
\end{aligned}
$$

represent the errors in the $x$-coordinate of the sensor position, the $y$-coordinate of the sensor position, and the TDOA measurement, respectively. The WMLS solution to (4), which ignores the effect of errors in the matrix $\mathbf{G}_{0}{ }^{3}$ is given by

$$
\widehat{\boldsymbol{u}}_{1}=\left(\mathbf{G}_{1}^{T} \mathbf{W}_{1} \mathbf{G}_{1}\right)^{-1} \mathbf{G}_{1}^{T} \mathbf{W}_{1} \mathbf{h}_{1}
$$

where the weighting matrix $\mathbf{W}_{1}$ is an estimate of the inverse of the autocorrelation matrix $E\left\{\left(\Delta \mathbf{h}_{1}-\right.\right.$ $\left.\left.\Delta \mathbf{G}_{1} \mathbf{u}_{0}\right)\left(\Delta \mathbf{h}_{1}-\Delta \mathbf{G}_{1} \mathbf{u}_{0}\right)^{T}\right\}$ under the assumption that there are no sensor errors and $\delta$ is a vector of i.i.d. zero-mean Gaussian random variables. ${ }^{4}$ The estimate given by (7) constitutes the output of Stage 1 of the WMLS algorithm.

For the second stage of the algorithm, the possible inconsistency between the estimated values for $\left(x_{0}, y_{0}\right)$ and $r_{0}=\sqrt{x_{0}^{2}+y_{0}^{2}}$ obtained in the vector $\hat{\boldsymbol{u}}_{1}$ is resolved by computing a new estimate of $\left(x_{0}^{2}, y_{0}^{2}\right)$ based on $\hat{\boldsymbol{u}}_{1}$. The approach is again weighted-least-squares, and we begin by defining

$$
\begin{aligned}
\mathbf{G}_{2} & =\left[\begin{array}{ll}
1 & 0 \\
0 & 1 \\
1 & 1
\end{array}\right], \quad \mathbf{h}_{2}=\hat{\boldsymbol{u}}_{1} \circ \hat{\boldsymbol{u}}_{1}=\left[\begin{array}{c}
\left(\hat{\boldsymbol{u}}_{1}(1)\right)^{2} \\
\left(\hat{\boldsymbol{u}}_{1}(2)\right)^{2} \\
\left(\hat{\boldsymbol{u}}_{1}(3)\right)^{2}
\end{array}\right], \\
\Delta \mathbf{h}_{2} & =\mathbf{h}_{2}-\mathbf{u}_{0} \circ \mathbf{u}_{0}=\mathbf{h}_{2}-\mathbf{G}_{2}\left[\begin{array}{c}
x_{0}^{2} \\
y_{0}^{2}
\end{array}\right],
\end{aligned}
$$

where the symbol " $\mathbf{u} \circ \mathbf{u}$ " indicates the Hadamard product of the vector $\mathbf{u}$ with the vector $\mathbf{v}$. It should be noted that the equation

$$
\mathbf{G}_{2}\left[\begin{array}{l}
x_{0}^{2} \\
y_{0}^{2}
\end{array}\right]=\mathbf{u}_{0} \circ \mathbf{u}_{0}
$$

is always satisfied, while the associated equation

$$
\mathbf{G}_{2}\left[\begin{array}{c}
\left(\hat{\boldsymbol{u}}_{1}(1)\right)^{2} \\
\left(\hat{\boldsymbol{u}}_{1}(2)\right)^{2}
\end{array}\right]=\mathbf{h}_{2}
$$

will not generally be satisfied. The estimate $\hat{\boldsymbol{u}}_{2}$ of $\left(x_{0}^{2}, y_{0}^{2}\right)$ is computed as a weighted least-squares solution to (10) and is given by

$$
\widehat{\boldsymbol{u}}_{2}=\left(\mathbf{G}_{2}^{T} \mathbf{W}_{2} \mathbf{G}_{2}\right)^{-1} \mathbf{G}_{2}^{T} \mathbf{W}_{2} \mathbf{h}_{2}
$$

where $\mathbf{W}_{2}$ is an estimate of the inverse of the autocorrelation matrix $E\left\{\Delta \mathbf{h}_{2} \Delta \mathbf{h}_{2}^{T}\right\}$. This estimate constitutes the output from Stage 2 of the algorithm.

The estimated values of $\left(x_{0}^{2}, y_{0}^{2}\right)$ obtained in the vector $\hat{\boldsymbol{u}}_{2}$ are used to obtain a final estimate of $\left(x_{0}, y_{0}\right)$ based on $\hat{\boldsymbol{u}}_{2}$ and $\hat{\boldsymbol{u}}_{1}$ combined. The final estimate $\hat{\boldsymbol{u}}_{3}$ of $\left(x_{0}, y_{0}\right)$ is given by

$$
\hat{\boldsymbol{u}}_{3}=\mathbf{P} \sqrt{\widehat{\boldsymbol{u}}_{2}}
$$

\footnotetext{
3 The explicit incorporation of sensor position errors in (4) will be utilized later to derive the WTLS algorithm and is also exploited in our expression for the bias of the WMLS algorithm. These errors have been ignored in previous analyses of this algorithm that have appeared in the literature.

${ }^{4}$ For example, for the simulations presented in this paper, the weighting matrix $\mathbf{W}_{1}$ is just a scaled version of the identity, which is a good approximation for $\left\{\left(\Delta \mathbf{h}_{1}-\Delta \mathbf{G}_{1} \mathbf{u}_{0}\right)\left(\Delta \mathbf{h}_{1}-\Delta \mathbf{G}_{1} \mathbf{u}_{0}\right)^{T}\right\}$ in the far field in the absence of sensor position errors.
} 
where, in this case, “ $\sqrt{\cdot}$ ” indicates a componentwise squareroot operation and $\mathbf{P}=\operatorname{diag}\left(\operatorname{sgn}\left(\hat{\boldsymbol{u}}_{1}(1)\right), \operatorname{sgn}\left(\hat{\boldsymbol{u}}_{1}(2)\right)\right)$. Details concerning the derivation of this algorithm can be found in $[8]$.

\subsection{Implementation of the WTLS algorithm}

To reduce the bias in the WMLS algorithm and to compensate somewhat for errors in sensor positions, a weighted total least-squares solution to (4) is used instead of the weighted minimum least-squares solution. To the best of our knowledge, such a weighted total least-squares algorithm has not been previously proposed in the literature. As such, this algorithm may be of interest in its own right.

To implement the algorithm, we define the matrices $\overline{\boldsymbol{G}}_{0}=$ $\left[\mathbf{G}_{0} \mid \mathbf{h}_{0}\right], \overline{\mathbf{G}}_{1}=\left[\mathbf{G}_{1} \mid \mathbf{h}_{1}\right]$ and $\bar{\Delta}_{1}=\left[\Delta \mathbf{G}_{1} \mid \Delta \mathbf{h}_{1}\right]$.

Note that (1) can now be rewritten as

$$
\overline{\mathbf{G}}_{0}\left[\frac{\mathbf{u}_{0}}{-1}\right]=0
$$

and (4) can be rewritten as

$$
\left(\overline{\boldsymbol{G}}_{1}-\bar{\Delta}_{1}\right)\left[\frac{\mathbf{u}_{0}}{-1}\right]=0 .
$$

In order to solve this system of equations, Cholesky decomposition is used. Let $\Sigma_{\mathrm{L}}=E\left\{\Delta_{1}^{T} \Delta_{1}\right\}$ and $\boldsymbol{\Sigma}_{\mathrm{R}}=E\left\{\Delta_{1} \Delta_{1}^{T}\right\}$ under the assumption that $\boldsymbol{\varepsilon}_{1}, \boldsymbol{\varepsilon}_{2}$, and $\boldsymbol{\delta}$ are mutually independent vectors of i.i.d. zero-mean Gaussian random variables. We seek a matrix $\bar{\Delta}$ and an estimate $\hat{\boldsymbol{u}}_{1}$ of $\mathbf{u}_{0}$ such that the equation

$$
\left(\overline{\mathbf{G}}_{1}-\bar{\Delta}\right)\left[\frac{\hat{\boldsymbol{u}}_{1}}{-1}\right]=0
$$

is satisfied, and the matrix $\bar{\Delta}$ has minimum possible norm of the form

$$
\|\bar{\Delta}\|=\sqrt{\operatorname{Tr}\left(\bar{\Delta}^{T} \Sigma_{\mathrm{R}}^{-1} \bar{\Delta} \boldsymbol{\Sigma}_{\mathrm{L}}^{-1}\right)} .
$$

To find $\hat{\boldsymbol{u}}_{1}$ and $\bar{\Delta}$, we first factor $\boldsymbol{\Sigma}_{\mathrm{L}}$ and $\boldsymbol{\Sigma}_{\mathrm{R}}$ using the Cholesky decomposition to get $\boldsymbol{\Sigma}_{\mathrm{L}}^{-1}=\mathbf{W}_{\mathrm{L}} \mathbf{W}_{\mathrm{L}}^{T}$ and $\boldsymbol{\Sigma}_{\mathrm{R}}^{-1}=\mathbf{W}_{\mathrm{R}} \mathbf{W}_{\mathrm{R}}^{T}$ and note that (15) can be rewritten as

$$
\mathbf{W}_{\mathrm{R}}^{T}\left(\overline{\boldsymbol{G}}_{1}-\bar{\Delta}\right) \mathbf{W}_{\mathrm{L}} \mathbf{W}_{\mathrm{L}}^{-1}\left[\frac{\hat{\boldsymbol{u}}_{1}}{-1}\right]=0
$$

or equivalently

$$
\left(\widetilde{\boldsymbol{G}}_{1}-\tilde{\Delta}\right) \tilde{\boldsymbol{u}}=0
$$

where

$$
\widetilde{\boldsymbol{G}}=\mathbf{W}_{\mathrm{R}}^{T} \overline{\boldsymbol{G}}_{1} \mathbf{W}_{\mathrm{L}}, \quad \tilde{\Delta}=\mathbf{W}_{\mathrm{R}}^{T} \bar{\Delta} \mathbf{W}_{\mathrm{L}}, \quad \tilde{\boldsymbol{u}}=\mathbf{W}_{\mathrm{L}}^{-1}\left[\frac{\hat{\boldsymbol{\mu}}_{1}}{-1}\right] .
$$

Now to solve (15), we look first for $\tilde{\Delta}$ and $\tilde{\boldsymbol{u}}$ that satisfy (18) such that $\bar{\Delta}$ has minimum possible Frobenius norm, which is given by

$$
\|\tilde{\Delta}\|_{\mathrm{F}}=\sqrt{\operatorname{Tr}\left(\tilde{\Delta}^{T} \tilde{\Delta}\right)}
$$

The desired solution for (18) is derived using singular value decomposition (SVD). Let the SVD of $\widetilde{\boldsymbol{G}}$ be given by

$$
\widetilde{\boldsymbol{G}}=\mathbf{U D V}^{T}
$$

where the columns of $\mathbf{V}$ are the orthonormal eigenvectors of $\widetilde{\boldsymbol{G}}^{T} \widetilde{\boldsymbol{G}}$. Let $\mathbf{v}_{\min }$ be the column of $\mathbf{V}$ corresponding to the smallest eigenvalue $\lambda_{\min }$ of $\widetilde{\boldsymbol{G}}^{T} \widetilde{\boldsymbol{G}}$. The desired solution to (18) is given by

$$
\tilde{\Delta}=\sqrt{\lambda_{\min }} \mathbf{v}_{\min } \mathbf{v}_{\min }^{T}, \quad \tilde{\boldsymbol{u}}=\alpha \mathbf{v}_{\min },
$$

where $\alpha$ is chosen such that

$$
\tilde{\boldsymbol{u}}=\alpha \mathbf{v}_{\min }=\mathbf{W}_{\mathrm{L}}^{-1}\left[\frac{\hat{\boldsymbol{u}}_{1}}{-1}\right]
$$

or equivalently

$$
\left[\frac{\hat{\boldsymbol{u}}_{1}}{-1}\right]=\alpha \mathbf{W}_{\mathrm{L}} \mathbf{v}_{\min }
$$

Hence, $\alpha$ is the negative of the inverse of the last component of the vector $\mathbf{W}_{\mathrm{L}} \mathbf{v}_{\text {min }}$, and the vector $\hat{\boldsymbol{u}}_{1}$ is the desired Stage 1 solution to the equation. Stage 2 and the final estimate for the WTLS algorithm are identical to the original Stage 2 and the final estimate for the WMLS algorithm.

It should be noted that the computational complexity of the WTLS algorithm is higher than that of the WMLS algorithm due solely to the computation of the SVD of an $M \times 4$ matrix. Unless the value of $M$ is extremely large, this represents a very slight increase in computational complexity.

\subsection{Implementation of the $\mathbf{N}-\mathrm{R}$ algorithm}

The Newton-Raphson method was implemented to solve the nonlinear TDOA equations iteratively by searching for the roots of the likelihood equation [21]. The WMLS estimate was used as a starting point for the iteration. Using the notation established above and assuming the target is in the far field and the errors in sensor positions are small relative to 
the target range, we can write

$$
\begin{aligned}
& d_{i}= \sqrt{\left(x_{0}-x_{i}\right)^{2}+\left(y_{0}-y_{i}\right)^{2}}-\sqrt{x_{0}^{2}+y_{0}^{2}}+c \delta_{i} \\
&= \sqrt{\left[x_{0}-\left(x_{i}+\varepsilon_{i 1}\right)+\varepsilon_{i 1}\right]^{2}+\left[y_{0}-\left(y_{i}+\varepsilon_{i 2}\right)+\varepsilon_{i 2}\right]^{2}} \\
&-\sqrt{x_{0}^{2}+y_{0}^{2}}+c \delta_{i} \\
&= \sqrt{\left[x_{0}-\hat{x}_{i}+\varepsilon_{i 1}\right]^{2}+\left[y_{0}-\hat{y}_{i}+\varepsilon_{i 2}\right]^{2}}-\sqrt{x_{0}^{2}+y_{0}^{2}}+c \delta_{i} \\
& \approx \sqrt{\left(x_{0}-\hat{x}_{i}\right)^{2}+\left(y_{0}-\hat{y}_{i}\right)^{2}}-\sqrt{x_{0}^{2}+y_{0}^{2}} \\
&+\frac{\left(x_{0}-\hat{x}_{i}\right) \varepsilon_{i 1}+\left(y_{0}-\hat{y}_{i}\right) \varepsilon_{i 2}}{\hat{r}_{i}}+c \delta_{i} \\
&= \sqrt{\left(x_{0}-\hat{x}_{i}\right)^{2}+\left(y_{0}-\hat{y}_{i}\right)^{2}}-\sqrt{x_{0}^{2}+y_{0}^{2}}+\xi_{i}+c \delta_{i}, \\
& i=1, \ldots, M,
\end{aligned}
$$

where $d_{i}=c \tau_{i}$ is the observed distance difference between the signal at receiver $(0,0)$ and the signal at receiver $\left(x_{i}, y_{i}\right)$, $\left(\hat{x}_{i}, \hat{y}_{i}\right)=\left(x_{i}, y_{i}\right)+\left(\varepsilon_{i 1}, \varepsilon_{i 2}\right)$ is the measured position of the $i$ th receiver, $\hat{r}_{i}$ is the range of target from the measured position of the $i$ th receiver, and

$$
\xi_{i}=\frac{\left(x_{0}-\hat{x}_{i}\right) \varepsilon_{i 1}+\left(y_{0}-\hat{y}_{i}\right) \varepsilon_{i 2}}{\hat{r}_{i}}
$$

represents the equivalent additive noise in the distancedifference measurement at the $i$ th receiver resulting from sensor position error. If we now let

$f\left(x_{0}, y_{0}\right)=\sum_{i=1}^{M}\left(d_{i}-\left[\sqrt{\left(x_{0}-\hat{x}_{i}\right)^{2}+\left(y_{0}-\hat{y}_{i}\right)^{2}}-\sqrt{x_{0}^{2}+y_{0}^{2}}\right]\right)^{2}$,

then, under the assumption that the random variables $\left(\xi_{i}+\right.$ $c \delta_{i}$ ), for $i=1, \ldots, M$, are i.i.d. zero-mean Gaussian, ${ }^{5}$ the likelihood equation for the target position $\mathbf{x} \triangleq\left(x_{0}, y_{0}\right)^{T}$ becomes

$$
\mathbf{f}(\mathbf{x}) \triangleq\left[\begin{array}{c}
\frac{\partial}{\partial x_{0}} f\left(x_{0}, y_{0}\right) \\
\frac{\partial}{\partial y_{0}} f\left(x_{0}, y_{0}\right)
\end{array}\right]=0
$$

\footnotetext{
${ }^{5}$ As a general rule, sensor position errors will be time-varying and well modeled as random only if sensor positions are estimated along with target position. In that case, the error in target location resulting from sensor position errors will not appear as a bias, but rather as an increase in the variance of the location error. Throughout this paper, we generally treat sensor position errors as fixed over time and represent the resulting target location error as a bias. Nevertheless, the CRLB including the effect of random sensor errors is a useful reference point that has been included in some of the simulation results presented in this paper.
}

Finally, letting

$$
\mathbf{J}(\mathbf{x}) \triangleq\left[\begin{array}{cc}
\frac{\partial^{2}}{\partial x_{0}^{2}} f\left(x_{0}, y_{0}\right) & \frac{\partial^{2}}{\partial x_{0} \partial y_{0}} f\left(x_{0}, y_{0}\right) \\
\frac{\partial^{2}}{\partial y_{0} \partial x_{0}} f\left(x_{0}, y_{0}\right) & \frac{\partial^{2}}{\partial y_{0}^{2}} f\left(x_{0}, y_{0}\right)
\end{array}\right],
$$

the Newton-Raphson iteration for the $(n+1)$ st estimate of the target position becomes

$$
\mathbf{x}_{n+1}=\mathbf{x}_{n}-\mathbf{J}^{-1}\left(\mathbf{x}_{n}\right) \mathbf{f}\left(\mathbf{x}_{n}\right)
$$

The output of the N-R algorithm is the result of this iteration given an appropriate stopping criterion.

If the initial location estimate for the N-R algorithm (i.e., the output from the WMLS algorithm) is reasonably accurate, the increase in computational complexity of the WMLS algorithm is minimal. In fact, although we did not perform a careful study of the convergence of the N-R algorithm, our simulation results indicate that in most cases, a significant performance improvement is achieved with a single step of the N-R iteration.

\section{PERFORMANCE EVALUATION RESULTS}

In this section, we present the results of a comprehensive performance evaluation of a 2D UWB-IR localization system. To establish the theoretical performance limits, we derived the CRLB for TDOA location estimation in the far field under the assumption that the aggregate measurement errors in distance-difference due to both sensor position errors and TDOA measurement errors were i.i.d. zero-mean Gaussian. In order to state this bound in terms of received SNR rather than the variance of the TDOA measurement error, we utilized the CRLB for TDOA estimation for known signals in AWGN as the assumed relationship between SNR and TDOA error variance under the assumption that the bandwidth of the UWB signal was $4 \mathrm{GHz}$.

To establish some practical performance limits and compare these with the theoretical limits, we utilized both analytical and numerical performance evaluation. The statistical characteristics (bias vector, autocorrelation matrix, total bias, and MSE) of the WMLS algorithm were derived analytically under the assumption that the sensor errors were deterministic and the TDOA measurement errors were i.i.d. zero-mean Gaussian. To validate these analytical results and compare the performance of the WMLS algorithm with both the new WTLS algorithm and the approximate ML estimate given by the N-R algorithm, we conducted an extensive Monte Carlo simulation study.

We begin by summarizing the analytical results on the statistical characteristics of the WMLS algorithm and the CRLB for TDOA location estimation. The details regarding the derivation of these results are presented in an appendix to this paper, which is available on request by contacting the author at e-mail; rbarton@uh.edu. 


\subsection{Analytical Results for WMLS Algorithm and CRLB}

Throughout the remainder of this paper, we make the assumption that the TDOA measurement error vector $\delta$ is a zero-mean Gaussian random vector with covariance matrix $\sigma_{\delta}^{2} \mathbf{I}$, where the variance $\sigma_{\delta}^{2}$ is related to the SNR of the received UWB signals by the formula (see, e.g., [1])

$$
\sigma_{\delta}^{2}=\frac{1}{8 \pi^{2} \operatorname{SNR}\left(16 \times 10^{18}\right)} \sec ^{2} .
$$

Under this assumption, the bias vector and autocorrelation matrix for the location estimates produced by the WMLS algorithm are given by

$$
\begin{aligned}
& \boldsymbol{\beta}=\left[\begin{array}{l}
\beta_{1} \\
\beta_{2}
\end{array}\right]=\left[\begin{array}{cc}
x_{0}^{-1} & 0 \\
0 & y_{0}^{-1}
\end{array}\right] \\
& \times\left(\left[\begin{array}{ccc}
x_{0}^{-1} & 0 & r_{0}^{-1} \\
0 & y_{0}^{-1} & r_{0}^{-1}
\end{array}\right] \mathbf{G}_{0}^{T} \mathbf{W}_{1} \mathbf{G}_{0}\left[\begin{array}{cc}
x_{0}^{-1} & 0 \\
0 & y_{0}^{-1} \\
r_{0}^{-1} & r_{0}^{-1}
\end{array}\right]\right)^{-1} \\
& \times\left[\begin{array}{ccc}
x_{0}^{-1} & 0 & r_{0}^{-1} \\
0 & y_{0}^{-1} & r_{0}^{-1}
\end{array}\right] \\
& \times\left(\mathbf { G } _ { 0 } ^ { T } \mathbf { W } _ { 1 } \left[c^{2} \sigma_{\delta}^{2}\left[\begin{array}{c}
1 \\
1 \\
\vdots \\
1
\end{array}\right]-\boldsymbol{\varepsilon}_{1} \circ \boldsymbol{\varepsilon}_{1}-\boldsymbol{\varepsilon}_{2} \circ \boldsymbol{\varepsilon}_{2}+2\left(x_{0}-\mathbf{x}\right) \circ \boldsymbol{\varepsilon}_{1}\right.\right. \\
& +2\left(y_{0}-\mathbf{y}\right) \circ \boldsymbol{\varepsilon}_{2}+4 c^{2} \sigma_{\delta}^{2} r_{0} \mathbf{W}_{1} \mathbf{G}_{0}\left(\mathbf{G}_{0}^{T} \mathbf{W}_{1} \mathbf{G}_{0}\right)^{-1}\left[\begin{array}{l}
0 \\
0 \\
1
\end{array}\right] \\
& +2\left(c^{2} \sigma_{\delta}^{2}\left[\begin{array}{c}
1 \\
1 \\
\vdots \\
1
\end{array}\right] \boldsymbol{\varepsilon}_{1}^{T}+2\left[\left(x_{0}-\mathbf{x}\right) \circ \boldsymbol{\varepsilon}_{1}\right] \boldsymbol{\varepsilon}_{1}^{T}\right. \\
& \left.+2\left[\left(y_{0}-\mathbf{y}\right) \circ \boldsymbol{\varepsilon}_{2}\right] \boldsymbol{\varepsilon}_{1}^{T}\right)^{T} \mathbf{W}_{1} \mathbf{G}_{0}\left(\mathbf{G}_{0}^{T} \mathbf{W}_{1} \mathbf{G}_{0}\right)^{-1}\left[\begin{array}{l}
1 \\
0 \\
0
\end{array}\right] \\
& +2\left(c^{2} \sigma_{\delta}^{2}\left[\begin{array}{c}
1 \\
1 \\
\vdots \\
1
\end{array}\right] \boldsymbol{\varepsilon}_{1}^{T}+2\left[\left(x_{0}-\mathbf{x}\right) \circ \boldsymbol{\varepsilon}_{1}\right] \boldsymbol{\varepsilon}_{1}^{T}\right.
\end{aligned}
$$$$
\left.\left.+2\left[\left(y_{0}-\mathbf{y}\right) \circ \boldsymbol{\varepsilon}_{2}\right] \boldsymbol{\varepsilon}_{1}^{T}\right)^{T} \mathbf{W}_{1} \mathbf{G}_{0}\left(\mathbf{G}_{0}^{T} \mathbf{W}_{1} \mathbf{G}_{0}\right)^{-1}\left[\begin{array}{l}
0 \\
1 \\
0
\end{array}\right]\right]
$$

$$
+2\left[\operatorname { T r } \left\{\left[\mathbf{W}_{1} \mathbf{G}_{0}\left(\mathbf{G}_{0}^{T} \mathbf{W}_{1} \mathbf{G}_{0}\right)^{-1} \mathbf{G}_{0}^{T}-\mathbf{I}\right]\right.\right.
$$$$
\times \mathbf{W}_{1}\left(c^{2} \sigma_{\delta}^{2}\left[\begin{array}{c}
1 \\
1 \\
\vdots \\
1
\end{array}\right] \varepsilon_{1}^{T}+2\left[\left(x_{0}-\mathbf{x}\right) \circ \boldsymbol{\varepsilon}_{1}\right] \boldsymbol{\varepsilon}_{1}^{T}\right.
$$$$
\left.\left.+2\left[\left(y_{0}-\mathbf{y}\right) \circ \boldsymbol{\varepsilon}_{2}\right] \boldsymbol{\varepsilon}_{1}^{T}\right)\right\}
$$$$
\times \operatorname{Tr}\left\{\left[\mathbf{W}_{1} \mathbf{G}_{0}\left(\mathbf{G}_{0}^{T} \mathbf{W}_{1} \mathbf{G}_{0}\right)^{-1} \mathbf{G}_{0}^{T}-\mathbf{I}\right]\right.
$$$$
\times \mathbf{W}_{1}\left(c^{2} \sigma_{\delta}^{2}\left[\begin{array}{c}
1 \\
1 \\
\vdots \\
1
\end{array}\right] \boldsymbol{\varepsilon}_{2}^{T}+2\left[\left(x_{0}-\mathbf{x}\right) \circ \boldsymbol{\varepsilon}_{1}\right] \boldsymbol{\varepsilon}_{2}^{T}\right.
$$$$
\left.\left.+2\left[\left(y_{0}-\mathbf{y}\right) \circ \boldsymbol{\varepsilon}_{2}\right] \boldsymbol{\varepsilon}_{2}^{T}\right)\right\}
$$$$
\left.\left.\times 2 c^{2} \sigma_{\delta}^{2} r_{0} \operatorname{Tr}\left\{\left[\mathbf{W}_{1} \mathbf{G}_{0}\left(\mathbf{G}_{0}^{T} \mathbf{W}_{1} \mathbf{G}_{0}\right)^{-1} \mathbf{G}_{0}^{T}-\mathbf{I}\right] \mathbf{W}_{1}\right\}\right]\right)
$$$$
\boldsymbol{\Sigma}=\left[\begin{array}{cc}
x_{0}^{-1} & 0 \\
0 & y_{0}^{-1}
\end{array}\right]\left(\left[\begin{array}{ccc}
x_{0}^{-1} & 0 & r_{0}^{-1} \\
0 & y_{0}^{-1} & r_{0}^{-1}
\end{array}\right] \mathbf{G}_{0}^{T} \mathbf{W}_{1} \mathbf{G}_{0}\left[\begin{array}{cc}
x_{0}^{-1} & 0 \\
0 & y_{0}^{-1} \\
r_{0}^{-1} & r_{0}^{-1}
\end{array}\right]\right)^{-1}
$$$$
\left(\left[\begin{array}{ccc}
x_{0}^{-1} & 0 & r_{0}^{-1} \\
0 & y_{0}^{-1} & r_{0}^{-1}
\end{array}\right] \mathbf{G}_{0}^{T} \mathbf{W}_{1} \mathbf{W}^{-1} \mathbf{W}_{1} \mathbf{G}_{0}\left[\begin{array}{cc}
x_{0}^{-1} & 0 \\
0 & y_{0}^{-1} \\
r_{0}^{-1} & r_{0}^{-1}
\end{array}\right]\right)
$$$$
\cdot\left(\left[\begin{array}{ccc}
x_{0}^{-1} & 0 & r_{0}^{-1} \\
0 & y_{0}^{-1} & r_{0}^{-1}
\end{array}\right] \mathbf{G}_{0}^{T} \mathbf{W}_{1} \mathbf{G}_{0}\left[\begin{array}{cc}
x_{0}^{-1} & 0 \\
0 & y_{0}^{-1} \\
r_{0}^{-1} & r_{0}^{-1}
\end{array}\right]\right)^{-1}\left[\begin{array}{cc}
x_{0}^{-1} & 0 \\
0 & y_{0}^{-1}
\end{array}\right]
$$ 
respectively, where $\mathbf{W}_{1}=\left(4 c^{2} \sigma_{\delta}^{2} r_{0}^{2}\right)^{-1} \mathbf{I}$ and

$$
\begin{aligned}
\mathbf{W}=\left(E\left\{\left(\Delta \mathbf{h}_{1}-\Delta \mathbf{G}_{1} \mathbf{u}_{0}\right)\left(\Delta \mathbf{h}_{1}-\Delta \mathbf{G}_{1} \mathbf{u}_{0}\right)^{T}\right\}\right)^{-1} \\
=\left[4 c^{2} \sigma_{\delta}^{2} r_{0}^{2} \mathbf{I}+4\left(\left(x_{0}-\mathbf{x}\right) \circ \boldsymbol{\varepsilon}_{1}+\left(y_{0}-\mathbf{y}\right) \circ \boldsymbol{\varepsilon}_{2}\right)\right. \\
\left.\quad \times\left(\left(x_{0}-\mathbf{x}\right) \circ \boldsymbol{\varepsilon}_{1}+\left(y_{0}-\mathbf{y}\right) \circ \boldsymbol{\varepsilon}_{2}\right)^{T}\right]^{-1} \\
=\frac{1}{4 c^{2} \sigma_{\delta}^{2} r_{0}^{2}} \cdot\left[\mathbf{I}-\left(\left(\left(x_{0}-\mathbf{x}\right) \circ \boldsymbol{\varepsilon}_{1}+\left(y_{0}-\mathbf{y}\right) \circ \boldsymbol{\varepsilon}_{2}\right)\right.\right. \\
\left.\quad \times\left(\left(x_{0}-\mathbf{x}\right) \circ \varepsilon_{1}+\left(y_{0}-\mathbf{y}\right) \circ \boldsymbol{\varepsilon}_{2}\right)^{T}\right) \\
\quad /\left(c^{2} \sigma_{\delta}^{2} r_{0}^{2}+\left(\left(x_{0}-\mathbf{x}\right) \circ \boldsymbol{\varepsilon}_{1}+\left(y_{0}-\mathbf{y}\right) \circ \boldsymbol{\varepsilon}_{2}\right)^{T}\right. \\
\left.\left.\quad \times\left(\left(x_{0}-\mathbf{x}\right) \circ \boldsymbol{\varepsilon}_{1}+\left(y_{0}-\mathbf{y}\right) \circ \boldsymbol{\varepsilon}_{2}\right)\right)\right] .
\end{aligned}
$$

The MSE for the algorithm is then given by $\varepsilon_{\mathrm{WMLS}}^{2}=\operatorname{Tr}(\Sigma)$, the total bias by $\beta_{\mathrm{WMLS}}=\|\beta\|_{2}=\sqrt{\beta_{1}^{2}+\beta_{1}^{2}}$, and the total variance by $\sigma_{\mathrm{WMLS}}^{2}=\mathcal{E}_{\mathrm{WMLS}}^{2}-\beta_{\mathrm{WMLS}}^{2}$.

Under the additional assumption that the aggregate sensor position error vector $\boldsymbol{\xi}=\left[\begin{array}{llll}\xi_{1} & \xi_{2} & \cdots & \xi_{M}\end{array}\right]^{T}$ is also a zero-mean Gaussian random vector with covariance matrix $\sigma_{\xi}^{2} \mathbf{I}$ that varies from estimate to estimate, (32) is reduced to

$$
\begin{aligned}
& \boldsymbol{\beta}=-2\left(c^{2} \sigma_{\delta}^{2}+\sigma_{\xi}^{2}\right)\left[\begin{array}{cc}
x_{0}^{-1} & 0 \\
0 & y_{0}^{-1}
\end{array}\right] \\
& \cdot\left(\left[\begin{array}{ccc}
x_{0}^{-1} & 0 & r_{0}^{-1} \\
0 & y_{0}^{-1} & r_{0}^{-1}
\end{array}\right] \hat{\boldsymbol{G}}_{0}^{T} \hat{\boldsymbol{G}}_{0}\left[\begin{array}{cc}
x_{0}^{-1} & 0 \\
0 & y_{0}^{-1} \\
r_{0}^{-1} & r_{0}^{-1}
\end{array}\right]\right)^{-1} \\
& \cdot\left[\begin{array}{ccc}
x_{0}^{-1} & 0 & r_{0}^{-1} \\
0 & y_{0}^{-1} & r_{0}^{-1}
\end{array}\right]\left[\begin{array}{c}
\sum_{i=1}^{M} \hat{x}_{i} \\
\sum_{i=1}^{M} \hat{y}_{i} \\
\sum_{i=1}^{M} c \hat{\tau}_{i}-2(4-M) r_{0}
\end{array}\right] \\
& \Sigma=4\left(c^{2} \sigma_{\delta}^{2}+\sigma_{\xi}^{2}\right) r_{0}^{2}\left[\begin{array}{cc}
x_{0}^{-1} & 0 \\
0 & y_{0}^{-1}
\end{array}\right] \\
& \times\left(\left[\begin{array}{ccc}
x_{0}^{-1} & 0 & r_{0}^{-1} \\
0 & y_{0}^{-1} & r_{0}^{-1}
\end{array}\right] \hat{\boldsymbol{G}}_{0}^{T} \hat{\boldsymbol{G}}_{0}\left[\begin{array}{cc}
x_{0}^{-1} & 0 \\
0 & y_{0}^{-1} \\
r_{0}^{-1} & r_{0}^{-1}
\end{array}\right]\right)^{-1}\left[\begin{array}{cc}
x_{0}^{-1} & 0 \\
0 & y_{0}^{-1}
\end{array}\right],
\end{aligned}
$$

respectively, where

$$
\begin{aligned}
\hat{\boldsymbol{G}}_{0} & =-2 \cdot\left[\begin{array}{ccc}
\hat{x}_{1} & \hat{y}_{1} & c \hat{\tau}_{1} \\
\hat{x}_{2} & \hat{y}_{2} & c \hat{\tau}_{2} \\
\vdots & \vdots & \vdots \\
\hat{x}_{M} & \hat{y}_{M} & c \hat{\tau}_{M}
\end{array}\right], \\
c \hat{\tau}_{i} & =\sqrt{\left(x_{0}-\hat{x}_{i}\right)^{2}+\left(y_{0}-\hat{y}_{i}\right)^{2}}-\sqrt{x_{0}^{2}+y_{0}^{2}}, \quad i=1, \ldots, M .
\end{aligned}
$$

Finally, if we make the additional simplifying assumption that the receiving sensors are nominally (i.e., ignoring sensor position error) arranged symmetrically in a circle of radius $r$ around the reference sensor at the origin, and we let $x_{0}=$ $r_{0} \cos \theta, y_{0}=r_{0} \sin \theta$, and

$$
\begin{aligned}
& \hat{x}_{i} \triangleq \hat{\rho}_{i} \cos \hat{\phi}_{i}=r \cos \hat{\phi}_{i}, \\
& \hat{y}_{i} \triangleq \hat{\rho}_{i} \sin \hat{\phi}_{i}=r \sin \hat{\phi}_{i}, \quad i=1,2, \ldots, M, \\
& \hat{v}_{i} \triangleq \sin \left(\theta-\hat{\phi}_{i}\right),
\end{aligned}
$$

then (34) can be simplified to give the following approximations for the total bias and the MSE:

$$
\begin{gathered}
\beta_{\mathrm{WMLS}}=\frac{4|4-M|\left(\sigma_{\xi}^{2}+c^{2} \sigma_{\delta}^{2}\right) r_{0}^{3}}{M r^{4} \bar{v}_{4}}, \\
\mathcal{E}_{\mathrm{WMLS}}^{2}=\frac{4\left(\sigma_{\xi}^{2}+c^{2} \sigma_{\delta}^{2}\right) r_{0}^{4}}{M r^{4} \bar{v}_{4}}
\end{gathered}
$$

where $\bar{v}_{4}=(1 / M) \sum_{i=1}^{M} \widehat{v}_{i}^{4}=(1 / M) \sum_{i=1}^{M} \sin ^{4}\left(\theta-\widehat{\phi}_{i}\right)$.

Similarly, under the assumption that $\boldsymbol{\xi}$ and $\boldsymbol{\delta}$ are zeromean Gaussian random vectors with covariance matrices $\sigma_{\xi}^{2} \mathbf{I}$ and $\sigma_{\delta}^{2} \mathbf{I}$, respectively, the CRLB for the variance of an unbiased location estimate derived from TDOA measurements in the far field is given by

$$
\mathrm{CRLB}_{\mathrm{TDOA}}=\frac{\left(\sigma_{\xi}^{2}+c^{2} \sigma_{\delta}^{2}\right) r_{0}^{2}\left(\|\tilde{\boldsymbol{x}}\|^{2}+\|\tilde{\boldsymbol{y}}\|^{2}\right)}{\|\tilde{\boldsymbol{x}}\|^{2}\|\tilde{\boldsymbol{y}}\|^{2}-|\langle\tilde{\boldsymbol{x}}, \tilde{\boldsymbol{y}}\rangle|^{2}},
$$

where

$$
\begin{aligned}
& \tilde{\boldsymbol{x}}=\left[\begin{array}{llll}
\hat{x}_{1}+c \hat{\tau}_{1} \cos \theta & \hat{x}_{2}+c \hat{\tau}_{2} \cos \theta & \ldots & \hat{x}_{M}+c \hat{\tau}_{M} \cos \theta
\end{array}\right]^{T}, \\
& \tilde{y}=\left[\begin{array}{llll}
\hat{y}_{1}+c \hat{\tau}_{1} \sin \theta & \hat{y}_{2}+c \hat{\tau}_{2} \sin \theta & \cdots & \hat{y}_{M}+c \hat{\tau}_{M} \sin \theta
\end{array}\right]^{T}, \\
& c \hat{\tau}_{i} \approx-\hat{\rho}_{i} \cos \left(\theta-\hat{\phi}_{i}\right)+\frac{\hat{\rho}_{i}^{2}}{2 r_{0}} \sin ^{2}\left(\theta-\hat{\phi}_{i}\right), \\
& \forall i=1,2, \ldots, M \text {. }
\end{aligned}
$$

If the sensors are nominally distributed uniformly in a circle of radius $r$ around the origin, this is reduced to

$$
\mathrm{CRLB}_{\mathrm{TDOA}}=\frac{4\left(\sigma_{\xi}^{2}+c^{2} \sigma_{\delta}^{2}\right) r_{0}^{4}}{M r^{4} \bar{v}_{4}}
$$

Notice that (38) and (41) imply that when the target is in the far field and the sensors are circularly symmetric with either no sensor position errors or Gaussian sensor position errors 
that vary independently from estimate to estimate, the MSE of the WMLS attains the CRLB for TDOA estimation. Unfortunately, as discussed previously when the sensor errors are fixed for long periods of time, the total bias of the algorithm is not insignificant in the far field and will not be averaged out by a tracker. Hence, the WMLS cannot be regarded as an approximately optimal algorithm in this case. The simulation results in the next section will demonstrate this quite clearly.

Notice also that the CRLB for TOA estimation in the far field with circularly symmetric sensors can be shown to be [22]

$$
\mathrm{CRLB}_{\mathrm{TOA}}=\frac{2\left(\sigma_{\xi}^{2}+c^{2} \sigma_{\delta}^{2}\right) r_{0}^{2}}{M r^{2}}
$$

Hence, we have

$$
\frac{\mathrm{CRLB}_{\mathrm{TDOA}}}{\mathrm{CRLB}_{\mathrm{TOA}}}=\frac{2 r_{0}^{2}}{\bar{v}_{4} r^{2}},
$$

so the lack of an absolute time reference for TDOA estimation results in an extremely significant performance penalty in the far field. Of course, this observation is valid for all location estimation systems, not just those based on UWB-IR signals, but the performance penalty is particularly significant when the goal is precision location estimation as it generally is with a UWB system.

\subsection{Simulation results}

The location estimation simulations were implemented using MATLAB. For most of the simulations, a total of nine receiver positions were used to compute the $2 \mathrm{D}$ location estimate of the target, with a reference sensor located at the origin and the remaining eight sensors positioned symmetrically around it in a circle of varying radius $r .{ }^{6}$ The relatively large number of sensors was selected in order to reduce the influence of target azimuth angle on performance to a negligible level. Sensor position errors relative to the reference sensor were simulated by adding i.i.d. AWGN samples with variance $\sigma_{\xi}^{2}$ to each of the true coordinate positions for the receivers located in the circular array around the reference sensor. Once the receiver and target positions were simulated, the relative distance differences were computed at the various receivers and translated into relative time differences. TDOA errors were simulated by adding i.i.d. AWGN samples with variance $\sigma_{\delta}^{2}$ to each of the computed relative time differences.

\subsubsection{One-shot WMLS performance}

To validate the analysis of the statistical performance characteristics for the WMLS algorithm derived in the appendix and summarized in Section 4.1 above, we conducted an initial set of simulations on the one-shot location estimation performance of the WMLS algorithm. Our analytical results

\footnotetext{
${ }^{6}$ Nine sensors were used in all simulation studies except for the study of performance versus number of receiving sensors.
}

indicate that, under the assumption that the target is in the far field and sensor errors are either absent or randomly distributed from estimate to estimate with a Gaussian distribution, the WMLS algorithm achieves the MSE of the best possible unbiased one-shot estimator. Thus these performance results also provide a good indication of the achievable accuracy of any stand-alone location estimation algorithm based on TDOA measurements of UWB-IR signals under similar system configurations. Accordingly, we have attempted to simulate a broad range of system configurations in order that these performance results may be used as a guideline for practical system design.

\section{Performance as a function of SNR}

The performance of the WMLS algorithm as a function of average received SNR is presented in Figure 1. For these plots, the radius of the receiver array was set to 10 meters and the target range to 100 meters, which corresponds to a range/baseline ratio of 10 . As the figure indicates, the simulated results agree very well with the analytical results. In the very low SNR region $(-30 \mathrm{~dB})$, the algorithm has an MSE of approximately $1000 \mathrm{~m}^{2}$ and a total bias of approximately 32 meters. In the high SNR region (10 dB), the algorithm has an MSE of approximately $0.079 \mathrm{~m}^{2}$ and a total bias of approximately 0.003 meters. These results indicate that under high SNR conditions, a one-shot TDOA UWB-IR localization system with $4 \mathrm{GHz}$ of bandwidth and a range/baseline radius of 10 can achieve a relative location error (i.e., root MSE over range) of approximately $0.28 \%$ at a range of 100 meters. Similarly, under very low SNR conditions, such a system can achieve a relative location error of only approximately $31.6 \%$.

\section{Performance as a function of range/baseline ratio}

The performance of the WMLS algorithm as a function of range/baseline ratio $\left(r_{0} / r\right)$ is presented in Figure 2. For these plots, the radius of the receiver array was fixed at 10 meters and the SNR was fixed at $-10 \mathrm{~dB}$. Once again, the simulation results are in good agreement with the analytical results. At this relatively low SNR level, an MSE of approximately $0.50 \mathrm{~m}^{2}$ and a total bias of approximately 0.04 meters are achievable with a range/baseline ratio of 5 . For a range/baseline ratio of 20, the MSE increases to approximately $125.9 \mathrm{~m}^{2}$ and the total bias increases to approximately 2.24 meters. In this case (baseline fixed at 10 meters), this corresponds to a relative location error of approximately $0.71 \%$ for a range/baseline ratio of 5 and a relative location error of approximately $11.2 \%$ for a range/baseline ratio of 20. As indicated in (38), if the range/baseline ratio remains fixed, the relative location error decreases linearly as range increases; whereas, for a fixed baseline radius, the relative location error increases linearly with increasing range.

\section{Performance as a function of number of receiver sensors}

The performance of the WMLS algorithm as a function of the number of sensors in the receiver array is presented in 


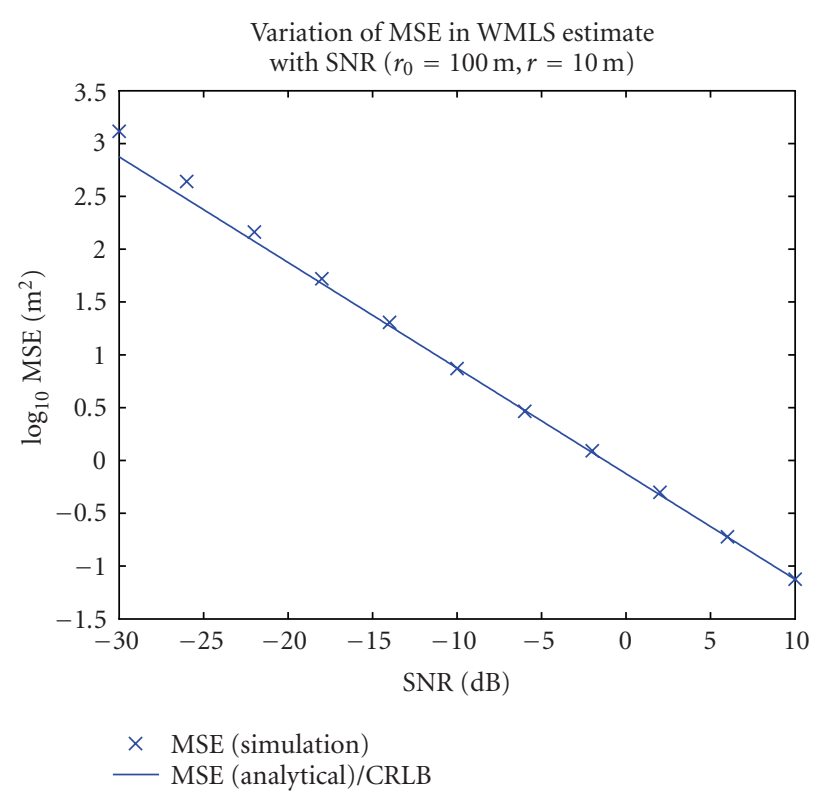

(a)

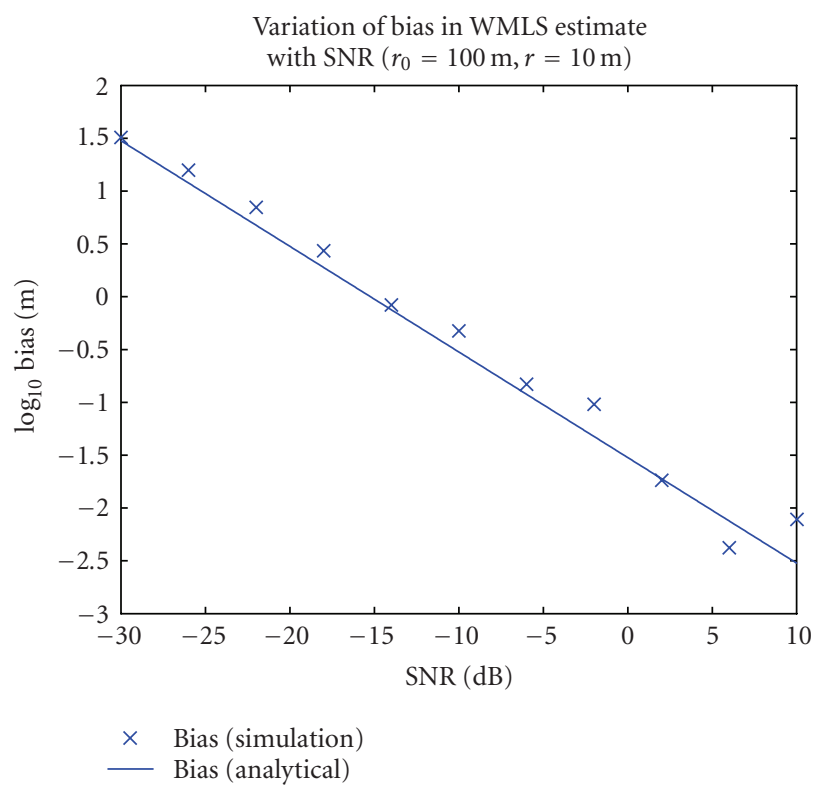

(b)

Figure 1: WMLS MSE (a) and total bias (b) as a function of SNR.

Figure 3. For these plots, the radius of the receiver array was fixed at 10 meters, the target range was fixed at 100 meters, and the SNR was fixed at $-10 \mathrm{~dB}$. The analytical results are again in good agreement with the simulated results with the exception of the case $M=4$. For this case, the analytical expression for the bias clearly understates the actual bias of the algorithm. This appears to result from the fact that some of the "negligible" terms dropped in the derivation of the analytical expression for the bias are in fact not negligible when $M=4$.

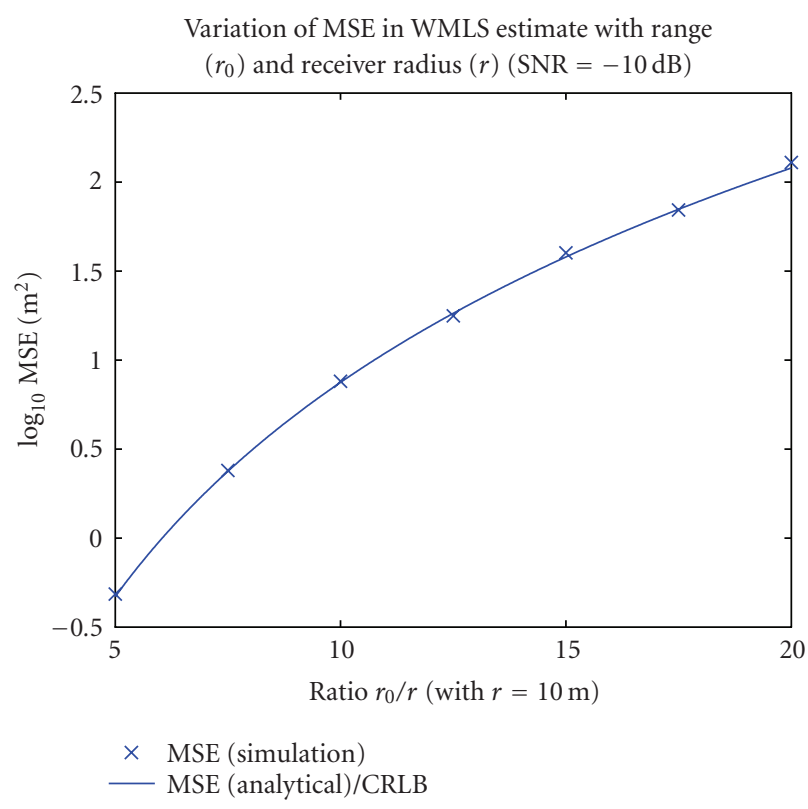

(a)

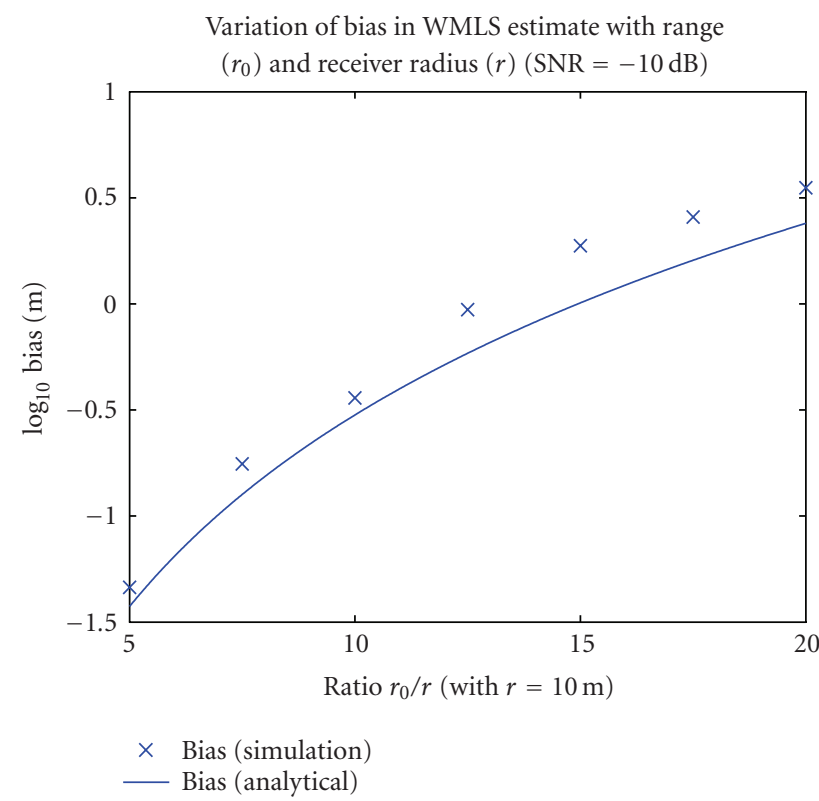

(b)

Figure 2: WMLS MSE (a) and total bias (b) as a function of range/baseline ratio.

As Figure 3 indicates, the achievable MSE with 4 receiving sensor is approximately $19.95 \mathrm{~m}^{2}$ and the achievable total bias is approximately 0.20 meters. With 11 receiving sensors, the achievable MSE decreases to approximately $6.17 \mathrm{~m}^{2}$ but the total bias increases to approximately 0.32 meters. Hence, with only 4 receiving sensors, a relative location error of approximately $4.46 \%$ can be achieved at 100 meters with a range/baseline radius of 10 and an SNR of $-10 \mathrm{~dB}$. With 11 receiving sensors, the relative location error can be reduced to $2.48 \%$. 


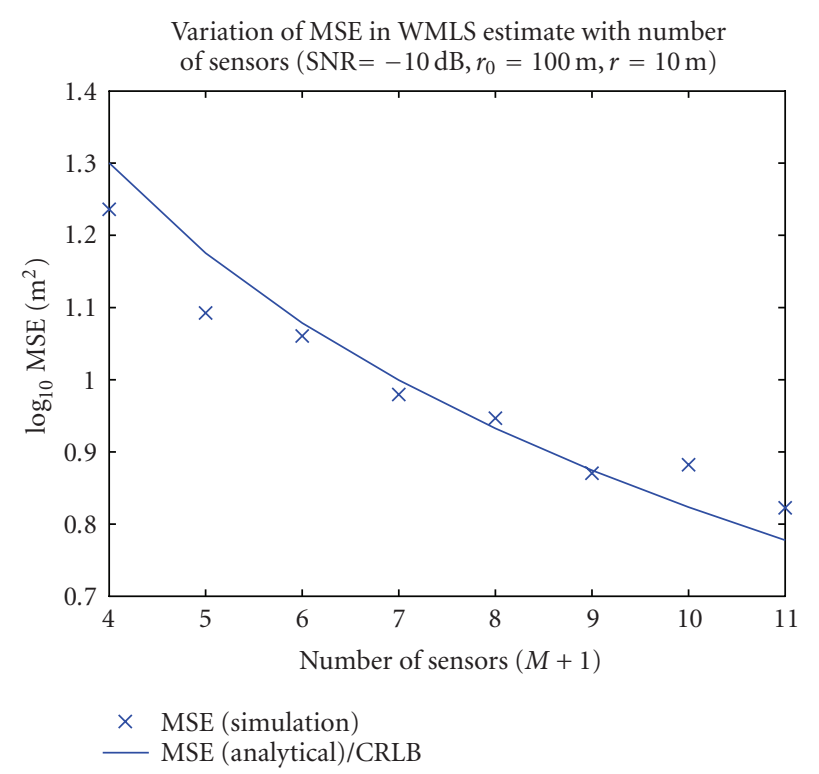

(a)

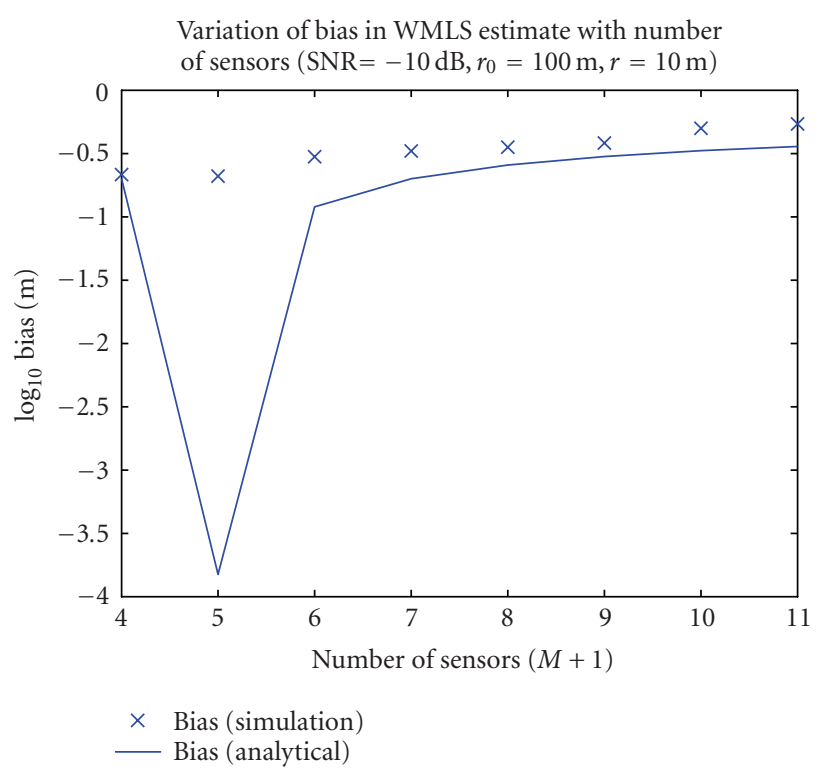

(b)

FIGURE 3: WMLS MSE (a) and total bias (b) as a function of number of receiving sensors.

\section{Performance as a function of sensor position error variance}

The performance of the WMLS algorithm as a function of the variance of random sensor position error is presented in Figure 4. Again for these plots, the radius of the receiver array was fixed at 10 meters, the target range was fixed at 100 meters, and the SNR was fixed at $-10 \mathrm{~dB}$. To reflect the fact that sensor errors will generally remain fixed over a large number of independent location estimates, the autocorrelation matrix and bias vector were estimated by averaging over a large number of independent trials for each set of randomly generated sensor errors before computing the MSE and total bias estimates that are plotted in Figure 4.

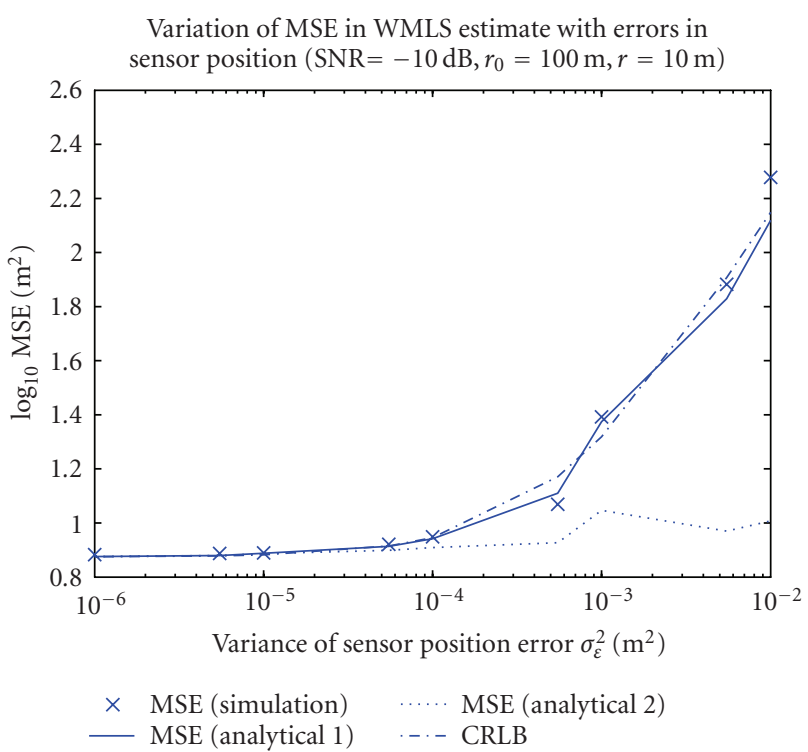

(a)

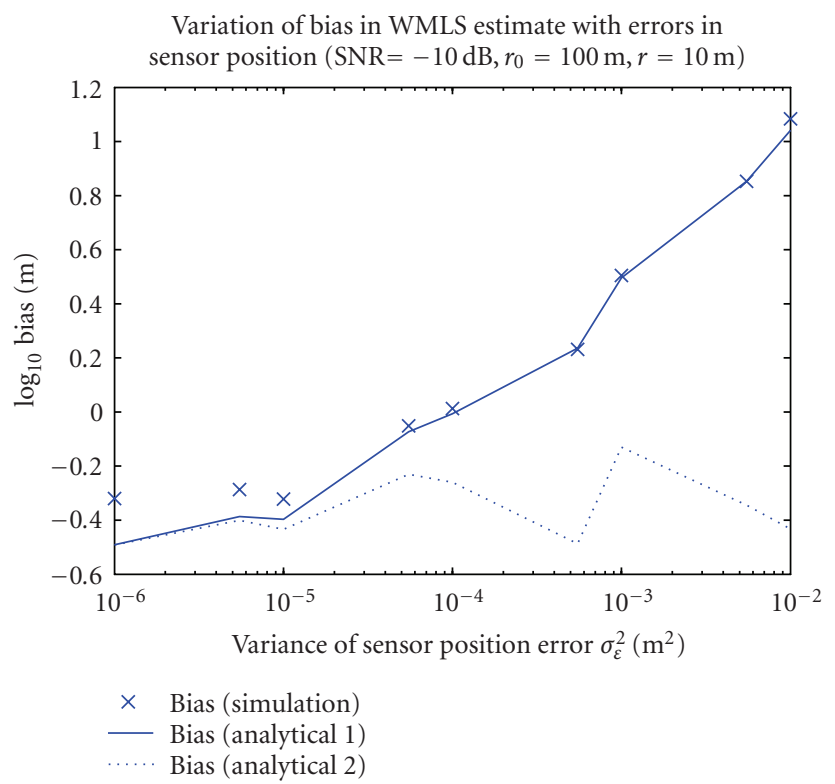

(b)

Figure 4: WMLS MSE (a) and total bias (b) as a function of sensor position error variance.

In this case, the plots show two different "analytical" curves: Analytical 1 and Analytical 2. The curves labeled Analytical 1 correspond to the MSE and bias predicted by (32), respectively, and these curves are in excellent agreement with the simulation results. The curves labeled Analytical 2, on the other hand, correspond to the MSE and bias predicted by (32) under the assumption that $\mathbf{W}_{1}=\mathbf{W}$. These curves represent the performance of a "genie-aided" version of the WMLS algorithm in which the weighting matrix $\mathbf{W}_{1}$ is computed using the true values of the sensor position errors.

There are several interesting aspects of the results presented in Figure 4. First, both the bias and MSE are fairly 
small in the low-sensor-noise regime where the dominant source of error is the TDOA measurement noise, but the bias (and consequently the MSE) increases dramatically as the sensor position errors become the dominant error mechanism. Second, the increase in bias due to the presence of sensor position errors can be effectively eliminated from the WMLS by using the "correct" version of the weighting matrix $\mathbf{W}_{1}$ in the algorithm. Although this is not actually feasible without modifying the algorithm extensively to estimate both sensor and target positions simultaneously, it does provide some insight into how the algorithm fails when sensor position errors are present and indicates how critical it is to account for possible sensor position errors in UWB location estimation algorithms.

Finally, the behavior of the CRLB relative to the MSE of the WMLS algorithm is of interest. First, notice that the CRLB curve does not always fall below either the simulated MSE or the Analytical 1 MSE curve. This is probably a result of the fact that the CRLB was derived assuming that the sensor position errors were i.i.d. zero-mean Gaussian that vary from estimate to estimate while the Analytical 1 curve and the simulated MSE were both computed by averaging over a relatively small number of random sensor position error vectors that were held fixed over a large number of independent location estimates. If the results had been averaged over a much larger number of random sensor errors (which would have required a much longer simulation time), both the Analytical 1 curve and the simulation results may well have fallen above the CRLB curve. ${ }^{7}$ Notice also that the bias of the WMLS estimate accounts for almost the entire MSE. Hence, while smoothing location estimates using a tracker will significantly reduce the CRLB, the bias of the WMLS will remain fixed and the MSE will remain correspondingly high. Finally, the fact that the CRLB, which corresponds to purely random sensor position errors that cannot be accurately determined, is much larger than the Analytical 2 MSE curve in the high-sensor-noise regime, which again indicates that it is essential to design UWB location estimation algorithms that are able to adapt to and compensate for the systematic sensor position errors that often occur in practice.

\subsubsection{Performance comparison of WMLS, WTLS, and $\mathrm{N}$-R algorithms}

In this subsection, we present simulation results comparing the performance of the WMLS algorithm with the new WTLS algorithm and the N-R algorithm. For these simulations, the baseline radius was fixed at 10 meters and the target range at 100 meters. Performance is compared both with and without the introduction of random sensor errors and both with and without averaging over multiple estimates.

\footnotetext{
${ }^{7}$ Recall, however, this is not a theoretical requirement since the CRLB is a lower bound only for the variance (and therefore the MSE) of unbiased estimates of location, while the WMLS has a significant bias.
}

\section{One-shot estimation with no errors in sensor position}

The performance of the three algorithms for one-shot location estimates with no errors in sensor position is presented in Figure 5. For these results, the SNR was varied across a very wide range from -10 to $30 \mathrm{~dB}$. As expected, the MSE of the WMLS algorithm is only slightly above the CRLB across the entire SNR range and approaches the CRLB asymptotically as the SNR becomes high. Further, as one might anticipate given the excellent MSE performance of the WMLS algorithm, the MSE for the (approximate maximumlikelihood) N-R algorithm is virtually identical to that of the WMLS algorithm, while the MSE of the WTLS algorithm is consistently (except for extremely low SNR) higher than both of the others. The degradation in MSE for the WTLS corresponds to a loss of approximately $1 \mathrm{~dB}$ in SNR across most of the SNR range.

Also as expected, the bias of both the WTLS and N-R algorithms is considerably lower than the bias of the WMLS algorithm for the entire range of SNR values. The reduction in bias seems to correspond to an average gain of approximately $5 \mathrm{~dB}$ in SNR across the SNR range. Note that for the WMLS algorithm, the bias dominates the MSE in the very low SNR regime but is negligible in the very high SNR regime so that the variance of the estimate dominates the MSE. For both the WTLS and N-R algorithms, the effect of the bias on the MSE is much less severe in the very low SNR regime.

\section{Block averaging with no errors in sensor position}

The performance of the three algorithms for smoothed estimates computed by averaging over a block of 100 consecutive one-shot estimates with no errors in sensor position is presented in Figure 6. As expected, the bias of the three algorithms has not changed as a result of the block averaging, but the MSE has decreased overall. These results demonstrate quite clearly the different manner in which the bias and variance of the one-shot location estimates affects the final smoothed estimate that would be produced by a tracker. In particular, in the very high SNR regime, where the error in the one-shot estimates is dominated by the variance of the estimates, the MSE of the averaged estimate is reduced by a factor of 100 for all three algorithms and approaches the CRLB. In contrast, the behavior in the very low SNR regime is quite different. In this case, the MSE of all three algorithms is far above the CRLB, but the MSE of the WMLS algorithm, which is dominated by the bias in this region, has not been reduced at all. On the other hand, the MSE of the WTLS and N-R algorithms, which are much closer to unbiased in this region, has been reduced by a factor of approximately 10 .

\section{One-shot estimation with errors in sensor position}

The performance of the three algorithms for one-shot location estimates with errors in sensor position is presented in Figure 7. For these results, the SNR was fixed at $-10 \mathrm{~dB}$ while 


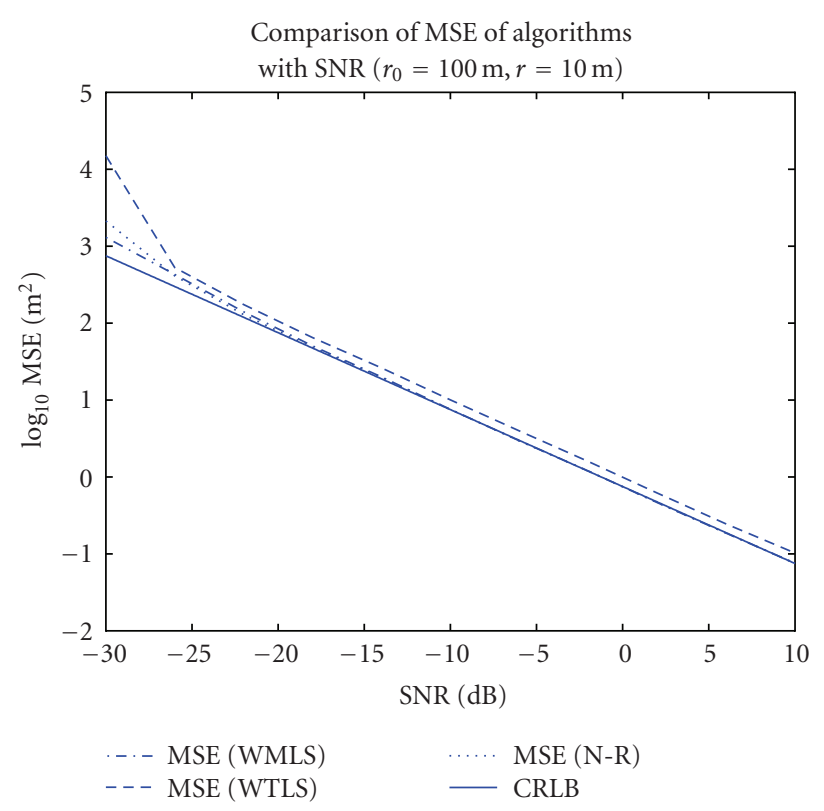

(a)

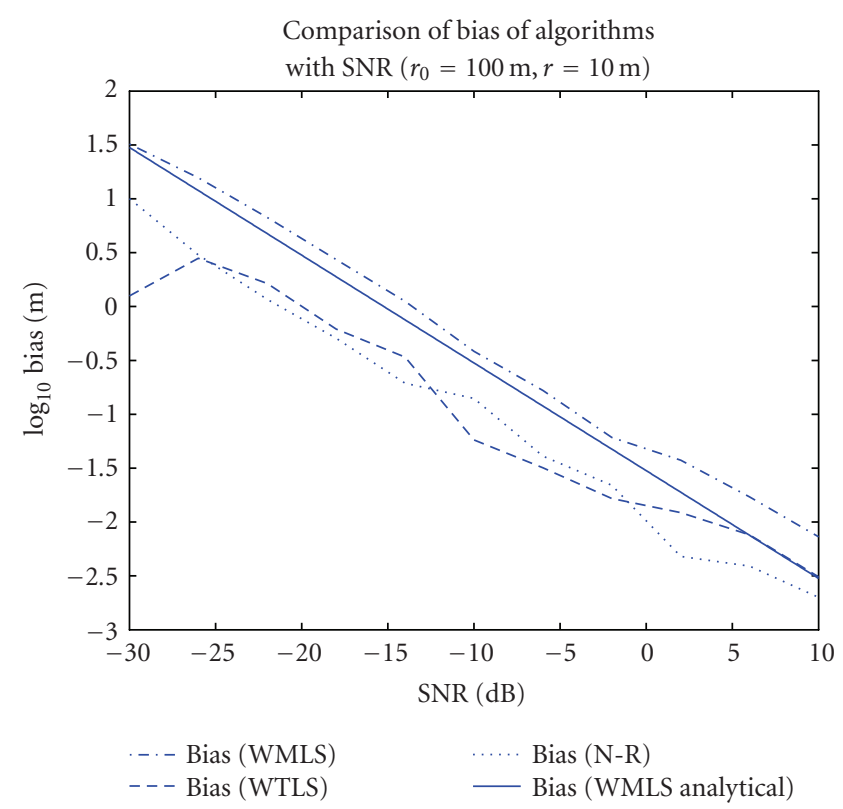

(b)

FIgURE 5: Comparison of MSE (a) and total bias (b) for WMLS, WTLS, and N-R algorithms for one-shot estimation with no sensor errors.

the variance of the sensor position noise was varied from $10^{-6} \mathrm{~m}^{2}$ to $10^{-2} \mathrm{~m}^{2}$. This range was chosen so that the error in the one-shot location estimates is dominated by TDOA errors at the low end of the range of sensor position error variance but dominated by sensor position errors at the high end. Note however, that even at the high end of the range, the standard deviation of the sensor positions from the nominal position on a circle of radius $10 \mathrm{~m}$ is only $1 \mathrm{~cm}$, which is still quite small.

These results demonstrate quite clearly the detrimental effects of even a small amount of sensor position error on

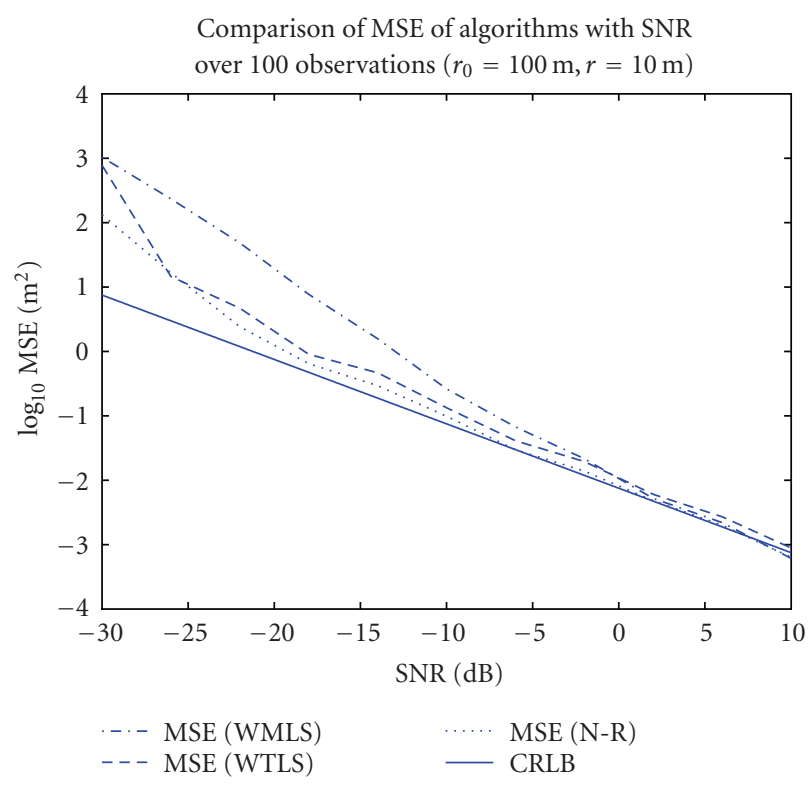

(a)

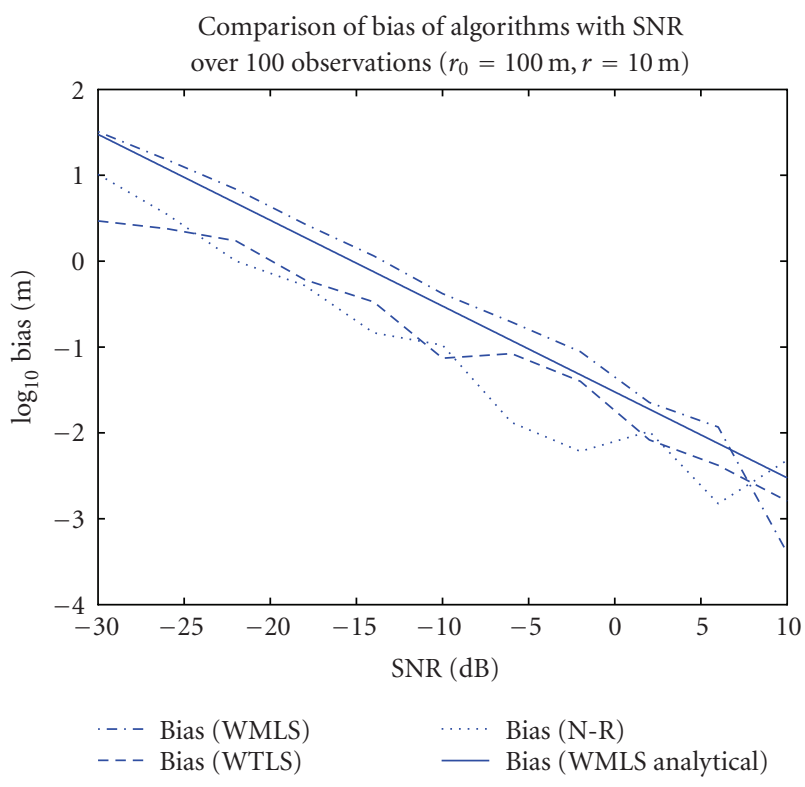

(b)

FIgure 6: Comparison of MSE (a) and total bias (b) for WMLS, WTLS, and N-R algorithms for smoothed estimation with no sensor errors.

the performance of UWB-IR location estimation algorithms. As the standard deviation of the sensor position error varies from $1 \mathrm{~mm}$ to only $1 \mathrm{~cm}$, the MSE in the location estimates for all of the algorithms studied here increases from approximately $3 \mathrm{~m}$ or $3 \%$ of the target range to almost $13 \mathrm{~m}$ or $13 \%$ of the target range. Furthermore, in the region where the estimation error is dominated by the sensor position error (above approximately $10 \mathrm{~mm}$ standard deviation of sensor position error), the MSE is dominated by the bias of the location estimates, which will not be reduced by the smoothing effects of a tracker. 


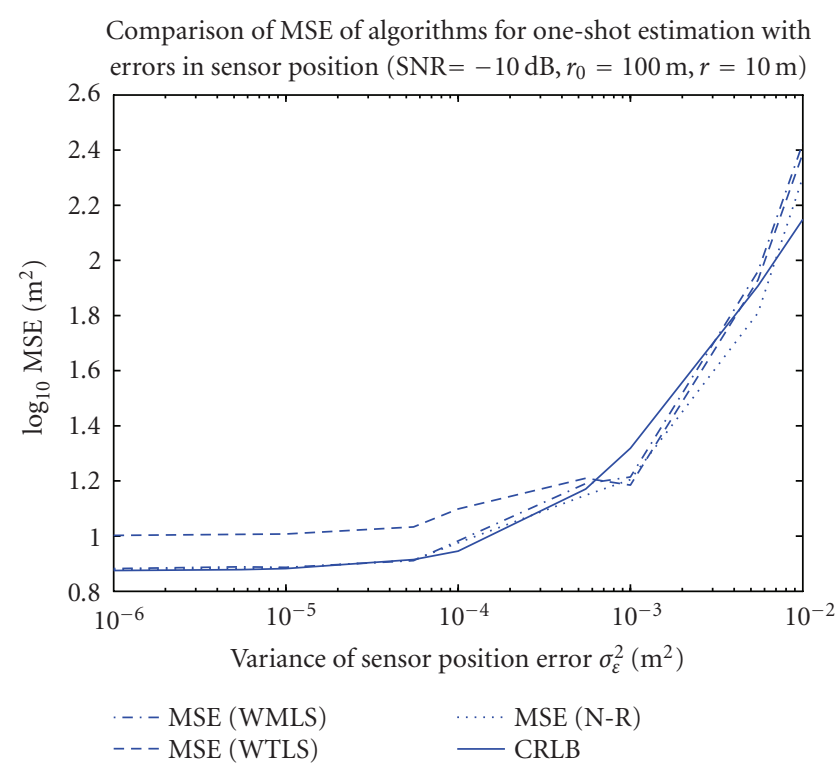

(a)

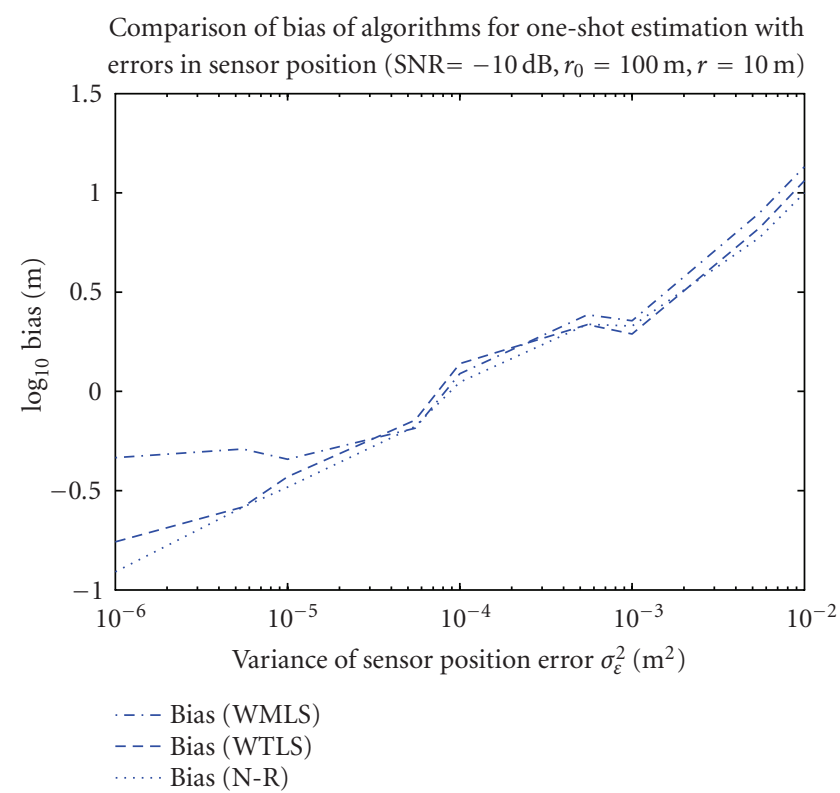

(b)

Figure 7: Comparison of MSE (a) and total bias (b) for WMLS, WTLS, and N-R algorithms for one-shot estimation with sensor errors.

\section{Block averaging with errors in sensor position}

The performance of the three algorithms for smoothed estimates computed by averaging over a block of 100 consecutive one-shot estimates with errors in sensor position is presented in Figure 8. Again, as expected, the bias of the three algorithms has not changed as a result of the block averaging, but in this case, the MSE has decreased significantly only in the region where the errors in location estimation are dominated by TDOA errors. In the region above there is approximately $1 \mathrm{~mm}$ of standard deviation in sensor position

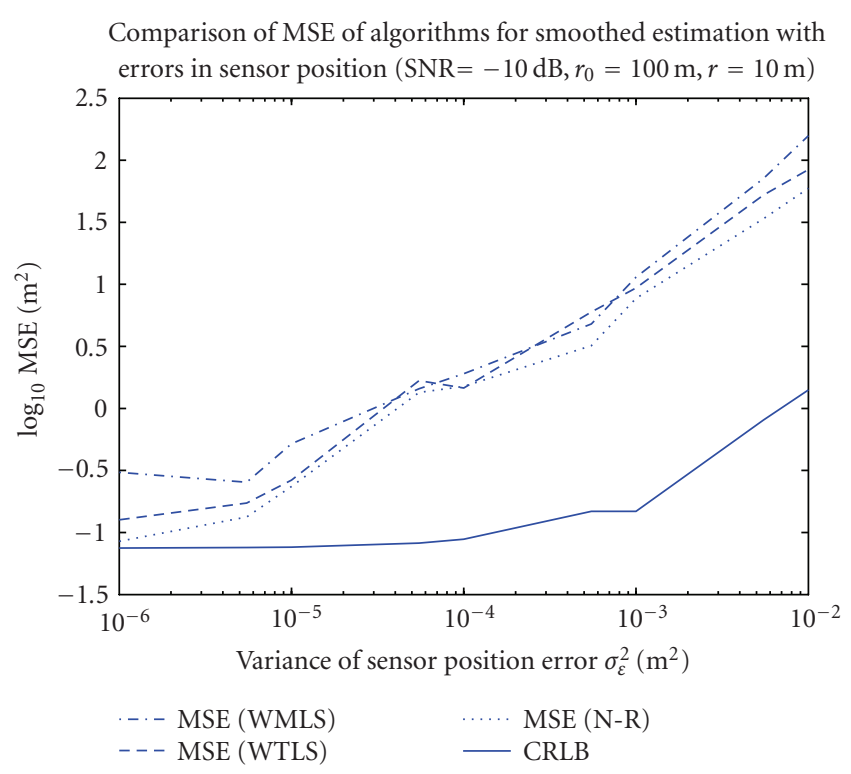

(a)

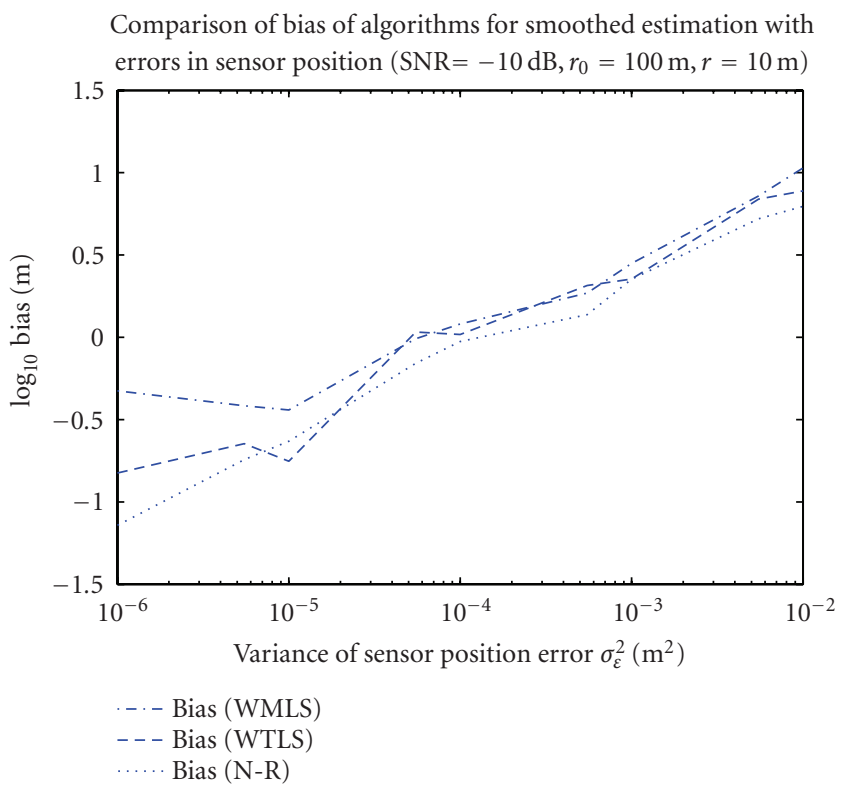

(b)

Figure 8: Comparison of MSE (a) and total bias (b) for WMLS, WTLS, and N-R algorithms for smoothed estimation with sensor errors.

error, the MSE has decreased very little for any of the algorithms. These results demonstrate even more dramatic impact of sensor position error on the performance of UWB-IR location estimation algorithms. In this case, as the standard deviation of the sensor position error varies from $1 \mathrm{~mm}$ to $1 \mathrm{~cm}$, the MSE in the location estimates for the WTLS and $\mathrm{N}-\mathrm{R}$ algorithms increases from approximately $30 \mathrm{~cm}$ or $0.3 \%$ of the target range to almost $10 \mathrm{~m}$ or $10 \%$ of the target range. The increase in MSE for the WMLS algorithm is not quite as dramatic, but only because the algorithm performs much worse in the presence of very low sensor position noise due 
to its increased bias. In all cases, the increase in MSE is quite dramatic for a very small increase in sensor position error.

\section{CONCLUSIONS}

In this paper, we have explored both the theoretical and practical performance limits of long-range UWB-IR TDOA location and tracking systems. The results presented in the paper demonstrate that such systems can deliver very accurate location estimates in the far field at reasonable SNR levels and should provide some valuable guidelines for practical system design. In addition, the results demonstrate that in order to achieve the high precision theoretically offered by such a system, one must utilize location estimation algorithms that are as nearly unbiased as possible in the absence of sensor position errors. We have derived and studied one such algorithm based on a novel constrained, weighted totalleast-squares approach and have shown that this algorithm as well as an approximate maximum-likelihood algorithm based on the Newton-Raphson method achieve an acceptably low bias with very little increase in computational complexity in comparison with a constrained, weighted minimumlease-squares algorithm widely studied in the literature.

Finally, the results demonstrate very clearly that relatively small errors in sensor position can completely undermine the potential utility of long-range UWB-IR location and tracking systems. As a practical consideration, such sensor position errors are probably unavoidable and must be adaptively estimated along with the target location in order to eliminate any systematic bias in the target location estimates.

\section{REFERENCES}

[1] S. Gezici, Z. Tian, G. B. Giannakis, et al., "Localization via ultra-wideband radios: a look at positioning aspects for future sensor networks," IEEE Signal Processing Magazine, vol. 22, no. 4, pp. 70-84, 2005.

[2] G. Schiavone, P. Wahid, E. Van Doom, R. Palaniappan, and J. Tracy, "Target detection and tracking using a UWB sensor web," in Proceedings of the IEEE Antennas and Propagation Society, International Symposium (AP-S '04), vol. 2, pp. 12871290, 2004.

[3] D. P. Young, C. M. Keller, D. W. Bliss, and K. W. Forsythe, "Ultra-wideband (UWB) transmitter location using time difference of arrival (TDOA) techniques," in Conference Record of the 37th Asilomar Conference on Signals, Systems, and Computers (ACSSC'03), vol. 2, pp. 1225-1229, Pacific Grove, Calif, USA, November 2003.

[4] K. Yu and I. Oppermann, "Performance of UWB position estimation based on time-of-arrival measurements," in Proceedings of the International Workshop on Ultra Wideband Systems; Joint with Conference on Ultra Wideband Systems and Technologies (UWBST-IWUWBS '04), pp. 400-404, Kyoto, Japan, 2004.

[5] FCC, "First notice and order: revision of part 15 of the commission's rules regarding ultra-wideband transmission systems," 2002.

[6] D. Dardari, C.-C. Chong, and M. Z. Win, "Improved lower bounds on time-of-arrival estimation error in realistic UWB channels," in Proceedings of the IEEE International Confer- ence on Ultra-Wideband, pp. 531-537, Waltham, Mass, USA, September 2006.

[7] B. M. Sadler, L. Huang, and Z. Xu, "Ziv-Zakai time delay estimation bound for ultra-wideband signals," in Proceedings of the IEEE International Conference on Acoustics, Speech, and Signal Processing (ICASSP '07), vol. 3, pp. 549-552, Honolulu, Hawaii, USA, April 2007.

[8] Y. T. Chan and K. C. Ho, "Simple and efficient estimator for hyperbolic location," IEEE Transactions on Signal Processing, vol. 42, no. 8, pp. 1905-1915, 1994.

[9] R. J. Barton, "Design and performance evaluation of a UWB communication and tracking system for mini-aercam," Final Report, NASA Johnson Space Center, Houston, Tex, USA, August 2005 .

[10] G. D. Arndt, E. T. Dickerson, and J. Ni, "Ultra-wideband twocluster angle-of-arrival tracking system design for space exploration," Tech. Rep., Institute for Space System Operations, University of Houston, University of Houston-Clear Lake, and NASA-Johnson Space Center, Houston, Tex, USA, 2006.

[11] C. Chang and A. Sahai, "Object tracking in a 2D UWB sensor network," in Conference Record of the 38th Asilomar Conference on Signals, Systems, and Computers (ACSSC '04), vol. 1, pp. 1252-1256, Pacific Grove, Calif, USA, November 2004.

[12] J. Zhang, R. A. Kennedy, and T. D. Abhayapala, "Cramér-Rao lower bounds for the time delay estimation of UWB signals," in Proceedings of the IEEE International Conference on Communications (ICC '04), vol. 6, pp. 3424-3428, Paris, France, June 2004.

[13] J. Zhang, R. A. Kennedy, and T. D. Abhayapala, "CramérRao lower bounds for the synchronization of UWB signals," EURASIP Journal on Applied Signal Processing, vol. 2005, no. 3, pp. 426-438, 2005.

[14] D. B. Jourdan, J. J. Deyst Jr., M. Z. Win, and N. Roy, "Monte Carlo localization in dense multipath environments using UWB ranging," in Proceedings of the IEEE International Conference on Ultra-Wideband (ICU '05), vol. 2005, pp. 314-319, Zurich, Switzerland, September 2005.

[15] B. T. Sieskul and T. Kaiser, "Cramér-Rao bound for TOA estimations in UWB positioning systems," in Proceedings of the IEEE International Conference on Ultra-Wideband (ICU '05), vol. 2005, pp. 408-413, Zurich, Switzerland, September 2005.

[16] X. Wang, Z. Wang, and B. O'Dea, “A TOA-based location algorithm reducing the errors due to non-line-of-sight (NLOS) propagation," IEEE Transactions on Vehicular Technology, vol. 52, no. 1, pp. 112-116, 2003.

[17] J. O. Smith and J. S. Abel, "Closed-form least-squares source location estimation from range-difference measurements," IEEE Transactions on Acoustics, Speech, and Signal Processing, vol. 35, no. 12, pp. 1661-1669, 1987.

[18] S. Van Huffel and J. Vandewalle, The Total Least Squares Problem: Computational Aspects and Analysis, SIAM, Philadelphia, Pa, USA, 1991.

[19] L. Kovavisaruch and K. C. Ho, "Alternate source and receiver location estimation using TDOA with receiver position uncertainties," in Proceedings of the IEEE International Conference on Acoustics, Speech, and Signal Processing (ICASSP '05), vol. IV, pp. 1065-1068, Philadelphia, Pa, USA, March 2005.

[20] L. Kovavisaruch and K. C. Ho, "Modified Taylor-series method for source and receiver localization using TDOA measurements with erroneous receiver positions," in Proceedings of the IEEE International Symposium on Circuits and Systems, vol. 3, pp. 2295-2298, Kobe, Japan, May 2005. 
[21] H. V. Poor, An Introduction to Signal Detection and Estimation, Springer, New York, NY, USA, 2nd edition, 1994.

[22] K. W. Cheung, H. C. So, W.-K. Ma, and Y. T. Chan, "Least squares algorithms for time-of-arrival-based mobile location," IEEE Transactions on Signal Processing, vol. 52, no. 4, pp. 11211128, 2004. 